\title{
Exit Time of a Hyperbolic $\alpha$-Stable Process from a Halfspace or a Ball
}

\author{
Michał Ryznar ${ }^{1}$ - Tomasz $\dot{Z}^{1} k^{1}$
}

Received: 11 August 2015 / Accepted: 14 January 2016 / Published online: 26 January 2016

(C) The Author(s) 2016. This article is published with open access at Springerlink.com

\begin{abstract}
For a hyperbolic $\alpha$-stable process in the hyperbolic space $\mathbb{H}^{d}, d \geq 2$, we prove that the mean exit time from a halfspace $H(a)=\left\{x_{d}>a\right\} \subset \mathbb{H}^{d}$ is equal to $\mathbb{E}^{x} \tau_{H(a)}=c(\alpha, d) \delta_{H(a)}^{\alpha / 2}(x)$, where $\delta_{D}(x)$ is the (hyperbolic) distance of $x$ to $D^{c}$. Based on this exact result we provide a sharp estimate of the mean exit time from a hyperbolic ball $B\left(x_{0}, R\right)$ of radius $R$ and center $x_{0}: \mathbb{E}^{x} \tau_{B\left(x_{0}, R\right)} \approx\left(\delta_{B\left(x_{0}, R\right)}(x) \tanh R\right)^{\alpha / 2}, x \in \mathbb{H}^{d}$. By usual isomorphism argument the same estimate holds in any other model of real hyperbolic space.
\end{abstract}

Keywords Hyperbolic Brownian motion $\cdot \alpha$-stable hyperbolic process · Transition density · Lévy measure · Exit time

Mathematics Subject Classification (2010) Primary 60J75 · Secondary 60J45

\section{Introduction}

Let $X=X(t), t \geq 0$, be a hyperbolic $\alpha$-stable process, $0<\alpha<2$, in the real hyperbolic space $\mathbb{H}^{d}, d \geq 2$, defined in a usual way as a hyperbolic Brownian motion subordinated to an independent $\alpha / 2$-stable subordinator. In the paper we compute the mean exit time from a halfspace $\left\{x \in \mathbb{H}^{d} ; x_{d}>a\right\}, a>0$, of the process $X$. As a function of $x=X(0)$ the mean exit time is, by definition, superharmonic in the underlying halfspace. The knowledge

The research was supported by NCN grant 2011/03/B/ST1/00423.

Michał Ryznar

michal.ryznar@pwr.edu.pl

Tomasz Żak

tomasz.zak@pwr.edu.pl

1 Faculty of Pure and Applied Mathematics, Wrocław University of Technology, ul. Wybrzeże Wyspiańskiego 27, 50-370 Wrocław, Poland 
of such function seems to be crucial in developing potential theory of hyperbolic $\alpha$-stable processes. For example, the recent progress in potential theory of subordinate Brownian motions or isotropic unimodal processes in $\mathbb{R}^{d}$ has one of its sources in applying the renewal function of the ladder height process of a one dimensional projection to construct a function which is harmonic in a halfspace. To some extent this compensates the lack of explicit formulas of Poisson kernels of balls or halfspaces.

Our derivation of the mean exit time of a halfspace is based on a connection between subordinate drifted Brownian motion (by standard $\alpha / 2$-stable subordinator) and relativistic stable process. Since exiting from a halfspace is completely described by the behaviour of the last coordinate of the process, actually the problem is one-dimensional and we take the advantage of the shape of the Green function of a halfline of relativistic process killed at some particular rate, found in [4].

Our main results are optimal estimates of the mean exit time from a hyperbolic ball of radius $R$. Let $\tau_{B\left(x_{0}, R\right)}$ be the first exit time from a ball of center $x_{0}$ and (hyperbolic) radius $R$. The estimates of the mean exit time when the process starts from the center of a ball, at least in the case when the radius $R>1$, were proved by Stós in [18] and the proof was based on the estimates of the transition density of the process. In this paper we extend those estimates to all starting points and radii. Our main result reads

$$
C_{1}\left(\delta_{B\left(x_{0}, R\right)}(x) \tanh R\right)^{\alpha / 2} \leq \mathbb{E}^{x} \tau_{B\left(x_{0}, R\right)} \leq C_{2}\left(\delta_{B\left(x_{0}, R\right)}(x) \tanh R\right)^{\alpha / 2}, \quad x \in \mathbb{H}^{d},
$$

where $\delta_{B\left(x_{0}, R\right)}(x)$ is the distance of $x$ to the complement of $B\left(x_{0}, R\right)$ and the constants $C_{1}$, $C_{2}$ depend only on $d$ and $\alpha$.

Here we mention that the same result holds for the hyperbolic Brownian motion with $\alpha=2$. Except for the case $d=3$ [16], we were unable to find a reference on that issue. Therefore we show how to obtain an exact solution for the mean exit time in terms of elementary functions (see Remark 3). The complexity of these solutions grows with the dimension and it seems difficult to use them in order to derive the estimates in general $\mathbb{H}^{d}$. On the other hand the estimates in general case are easy to obtain via Dynkin's lemma as presented in Remark 4.

Note that for small radii, say $R<1$, we have

$$
\mathbb{E}^{x} \tau_{B\left(x_{0}, R\right)} \approx\left(\delta_{B\left(x_{0}, R\right)}(x) R\right)^{\alpha / 2}, \quad x \in \mathbb{H}^{d} .
$$

Here $\approx$ means that both sides are comparable i.e. their ratio is bounded between two positive constants which depend only on $d$ and $\alpha$. By the result of Getoor [8] the mean exit time from Euclidean ball of radius $R$ for the isotropic stable process in $\mathbb{R}^{d}$ is equal to $C(\alpha, d)\left(R^{2}-|x|^{2}\right)^{\alpha / 2},|x| \leq R$. Hence the estimate in the hyperbolic case for small radii is of the same order as the mean exit time for the isotropic stable process. However for large radii, say $R \geq 1$, such comparison does not hold since the mean exit time in $\mathbb{H}^{d}$ is of order $\delta_{B\left(x_{0}, R\right)}^{\alpha / 2}(x)$. This is not surprising, since due to the drift, exiting from a large ball is comparable on average to exiting from a halfspace. In order to estimate the mean exit time from a ball, if the process starts from $x$ arbitrary close to the boundary of the ball, we take advantage of the hyperbolic geometry and invariance of the process with respect to hyperbolic isometries. This enables us to assume that $x_{0}=(0, \ldots, 0,1)$ and the starting point is of the form $x=\left(0, \ldots, 0, x_{d}\right)$. The upper bound is then easily obtained by observing that the ball is contained in a tangent strip and using the one-dimensional result. The lower bound is much more difficult to derive. We use a method which was applied in [1], where the corresponding estimate was found for a wide class of isotropic unimodal Lévy processes in $\mathbb{R}^{d}$. The method relies first on delicate estimates of the Dynkin-type generator applied to test functions which are constructed from the harmonic functions of 
halfspaces. In the second step a maximum principle is used. Here we follow this path and due to the first part of the paper we can use the calculated superharmonic function (the mean exit time from a halfspace) to construct the test function for the Dynkin-type generator of the hyperbolic stable process. Again our computations are substantially simplified by the proper usage of isometries of the hyperbolic space. Once the estimates of the action of the Dynkin type generator are established, the lower bound of the mean exit time is obtained by applying the maximum principle.

In a number of papers $[5,14,15]$, where potential theory of subordinate Brownian motions in real Euclidean spaces was developed, one of the main technical tools were estimates of the infinitesimal generator of the process on suitable sufficiently smooth functions (belonging to the domain of the generator) usually dependent on the distance to the complement of a given set. Such precise estimates via Dynkin lemma lead to sharp estimates of exit probabilities or Green functions. An advantage of our approach is that we do not require any assumptions on a measurable non-negative test function to apply a Dynkin-type generator. Moreover, some computations associated with Dynkin-type generator seem to be simpler than computations involving the infinitesimal generator.

There are heuristic arguments that the potential theory of hyperbolic stable process should be similar to the corresponding theory in the classical $d$-dimensional space. The local equivalence of the hyperbolic and Euclidean metrics together with the local comparability of hyperbolic and Euclidean Brownian motions are the sources of such claims, but we are not aware of any rigorous justifications. Our result can be regarded as an initial step towards showing that indeed the classical and hyperbolic stable processes have similar local properties. We conjecture for example that the Green functions are comparable for small balls or even for more general bounded sets with smooth boundaries. On the other hand in the large scale these two processes behave differently and finding large scale properties (e.g. a behaviour of a Green function of large sets, etc.) requires further exploration.

The paper is composed as follows. In Section 2 we introduce the hyperbolic space $\mathbb{H}^{d}$ and recall basic properties of the hyperbolic Brownian motion. In Section 3 we define the hyperbolic stable motion by usual procedure of subordination (see also [7]). Also some estimates related to the process are recalled. In Section 4 we derive an explicit formula of the mean exit time from a halfspace together with estimates of mean exit time from a strip, which are crucial for the next section. Section 5 is devoted to proving our main result which is the sharp estimate of the mean exit time from a ball. The most technical part of the paper is postponed to the last section, where delicate estimates of the Dynkin-type generator applied to appropriate test functions are obtained.

In the paper all constants, usually denoted by $c$ or $C$, are positive and depend only on $\alpha$ and $d$. Their values may change from line to line.

\section{Hyperbolic Space and Hyperbolic Brownian Motion}

Let us define $\mathbb{H}^{d}=\left\{x \in \mathbb{R}^{d}: x_{d}>0\right\}$. This half-space of $\mathbb{R}^{d}$, equipped with the Riemannian metric $d s^{2}=\frac{d x_{1}^{2}+\ldots+d x_{d}^{2}}{x_{d}^{2}}$, is a model of $d$-dimensional hyperbolic space. The volume element is given by $d \mu=\frac{d x_{1} \ldots d x_{d}}{x_{d}^{d}}$ and the hyperbolic distance $d_{\mathbb{H}^{d}}(x, y)$ is given by

$$
\cosh \left(d_{\mathbb{H}^{d}}(x, y)\right)=1+\frac{|x-y|^{2}}{2 x_{d} y_{d}},
$$


where $|x-y|$ denotes the Euclidean distance. Since $\mathbb{H}^{d}$ is a subset of $\mathbb{R}^{d}$, sometimes we will consider this set equipped with the Euclidean metric. In order to simplify notation and avoid misunderstanding, for $x, y \in \mathbb{H}^{d}$ we will denote by $|x-y|$ their Euclidean distance and by $d_{\mathbb{H} d}(x, y)$ their hyperbolic distance.

From the formula (1) it is easy to see that a hyperbolic ball with radius $R$ in $\mathbb{H}^{d}$ is also a Euclidean ball but with different center and radius. In particular, a hyperbolic ball $B((0, . ., 0,1), R)$ is a Euclidean ball with center $(0, \ldots, 0, \cosh R)$ and radius $\sinh R$.

For one-dimensional space $\mathbb{H}^{1}$ formula (1) gives the following: for $x, y \in \mathbb{H}^{1}$ (that is $x, y>0)$

$$
d_{\mathbb{H}^{1}}(x, y)=\left|\log \frac{x}{y}\right|,
$$

hence an open ball $B(1, R) \subset \mathbb{H}^{1}$ is an interval $\left(e^{-R}, e^{R}\right)$. Moreover, for points $x=$ $\left(0, \ldots, 0, x_{d-k+1}, . ., x_{d}\right)$ and $y=\left(0, \ldots, 0, y_{d-k+1}, . ., y_{d}\right), k \leq d$, their distance in $\mathbb{H}^{d}$ given by Eq. 1 is equal to their distance as points from $\mathbb{H}^{k}$.

The group of isometries of $\mathbb{H}^{d}$ is generated by Euclidean translations parallel to the boundary of $\mathbb{H}^{d}$, symmetries with respect to hyperplanes perpendicular to the boundary of $\mathbb{H}^{d}$ and by inversions of $\mathbb{H}^{d}$ with respect to half-spheres with centers on the boundary of $\mathbb{H}^{d}$ (see e.g. [17]). In particular, for any two points $A, B$ on a hyperbolic sphere $S\left(x_{0}, R\right)=\left\{x \in \mathbb{H}^{d}: d_{\mathbb{H} d}\left(x, x_{0}\right)=R\right\}$ there exists a hyperbolic isometry $I_{A B}$ such that $I_{A B}\left(S\left(x_{0}, R\right)\right)=S\left(x_{0}, R\right)$ and $I_{A B}(A)=B$. Next we may translate $S\left(x_{0}, R\right)$ such that its center $x_{0}$ has new coordinates $\left(0, \ldots, 0,\left(x_{0}\right)_{d}\right)$. The above-mentioned transformation are in general given by complicated formulas. In a very particular case, i.e. for $\mathbb{H}^{2}$, identified for simplicity of notation with $\{z \in \mathbb{C}: \Im z>0\}$, the hyperbolic rotation by angle $\theta$ around $i$ is given by the following simple formula

$$
I_{\theta}(z)=\frac{z \cos \frac{\theta}{2}+\sin \frac{\theta}{2}}{-z \sin \frac{\theta}{2}+\cos \frac{\theta}{2}} .
$$

Combinig hyperbolic isometries described above, we get the following fact, crucial for our future considerations:

Proposition 2.1 Any hyperbolic ball $B\left(x_{0}, R\right) \subset \mathbb{H}^{d}$ can be isometrically transformed onto a ball with center $(0, \ldots, 0,1)$ and radius $R$. Moreover, if $y$ is any fixed point of a sphere $S\left(x_{0}, R\right)$ then there exists a hyperbolic isometry $I$ such that $I\left(x_{0}\right)=(0, \ldots, 0,1)$ and $I(y)=\left(0, \ldots, 0, e^{-R}\right)$.

The Laplace-Beltrami operator in $\mathbb{H}^{d}$ is given by the formula

$$
\Delta_{L B}=x_{d}^{2}\left(\sum_{k=1}^{d} \frac{\partial^{2}}{\partial x_{k}^{2}}\right)-(d-2) x_{d} \frac{\partial}{\partial x_{d}} .
$$

This operator commutes with all isometries of $\mathbb{H}^{d}$ : if $I$ is such isometry then for all $f \in C^{2}\left(\mathbb{H}^{d}\right)$ there holds

$$
\Delta_{L B}(f \circ I)=\left(\Delta_{L B} f\right) \circ I .
$$

A hyperbolic Brownian motion $B(t)$ in $\mathbb{H}^{d}$ is a strong Markov process generated by $\Delta_{L B}$. Because $\Delta_{L B}$ commutes with isometries of $\mathbb{H}^{d}$, hyperbolic Brownian motion $B(t)$ starting from $x \in \mathbb{H}^{d}$ is invariant with respect to all hyperbolic isometries, leaving point $x$ invariant (similarly, as Brownian motion starting from $x \in \mathbb{R}^{d}$ is invariant with respect to all Euclidean isometries of $\mathbb{R}^{d}$, leaving the point $x$ invariant). To avoid misunderstanding we will denote Euclidean Brownian motions (with values in $\mathbb{R}$ or in $\mathbb{R}^{d}$ ) by $W(t)$. 
Observe that the part of $\Delta_{L B}$ that involves the first-order derivative acts only on the last coordinate, hence for twice differentiable $f$ depending only on $x_{d}$

$$
\Delta_{L B} f\left(x_{d}\right)=x_{d}^{2} f^{\prime \prime}\left(x_{d}\right)-(d-2) x_{d} f^{\prime}\left(x_{d}\right) .
$$

This means that the last coordinate of the hyperbolic Brownian motion in $\mathbb{H}^{d}$ is the following:

$$
B_{d}(t)=B_{d}(0) e^{W(t)-(d-1) t},
$$

where $W(t)$ is a one-dimensional real Wiener process with $\operatorname{Var}(W(t))=2 t$.

The hyperbolic Brownian motion in $\mathbb{H}^{d}$ has a transition density (with respect to the Riemannian measure $d \mu$ ) that depends on the hyperbolic distance between $x$ and $y$ : $g^{(d)}(t, x, y)=g^{(d)}\left(t, d_{\mathbb{H}^{d}}(y, x)\right)$. For simplicity, let us denote $d_{\mathbb{H}^{d}}(y, x)=r$. For all $d=1,2, \ldots$ the following Millson formula [10] holds

$$
g^{(d+2)}(t, r)=\frac{-e^{-(d-1) / 2}}{2 \pi \sinh r} \frac{\partial}{\partial r} g^{(d)}(t, r) .
$$

One can easily see from the form of Laplace-Beltrami operator in $\mathbb{H}^{1}$ that the one dimensional hyperbolic Brownian motion is the exponential of one dimensional Wiener process. Its density (with respect to $d \mu=\frac{d r}{r}$ ) for $r>0$ and $t>0$ is given by

$$
g^{(1)}(t, r)=\frac{1}{(4 \pi t)^{1 / 2}} e^{-r^{2} /(4 t)} .
$$

By the Millson formula, we conclude that for odd dimensions $d=1,3,5, \ldots$ this density is given by elementary functions (compare [10]). For $d=2$ the transition density of the hyperbolic Brownian motion is non-elementary:

$$
g^{(2)}(t, r)=\frac{\sqrt{2} e^{-t / 4}}{(4 \pi t)^{3 / 2}} \int_{r}^{\infty} \frac{s e^{-s^{2} /(4 t)}}{(\cosh s-\cosh r)^{1 / 2}} d s
$$

and this is also the case for all even dimensions $d$.

We are interested in processes, killed on exiting a set $D$. Let $X(t)$ be a stochastic process and let $D$ be an open set in $\mathbb{H}^{d}$. We define the exit time of $X$ from $D$ as

$$
\tau_{D}=\inf \{t: X(t) \notin D\},
$$

and the process killed on exiting $D$ by

$$
X^{D}(t)= \begin{cases}X(t), & t<\tau_{D}, \\ \delta, & t \geq \tau_{D},\end{cases}
$$

where $\delta$ is a 'cemetery point', i.e. some isolated point. If $X(t)$ has a transition density, then the killed process also has the transition density, given by Hunt's formula [12]:

$$
p^{D}(t, x, y)=p(t, x, y)-\mathbb{E}^{x}\left[\tau_{D}<t, p\left(t-\tau_{D}, X\left(\tau_{D}\right), y\right)\right] .
$$

We call $p^{D}(t, x, y)$ the Dirichlet heat kernel of $X$ on $D$.

We will also use $G_{D}(x, y)$, the Green function of $X$ on $D$. It is given by the formula

$$
G_{D}(x, y)=\int_{0}^{\infty} p^{D}(t, x, y) d t,
$$

whenever the integral is convergent. 


\section{Hyperbolic $\alpha$-Stable Process and its Lévy Measure}

In what follows, unless stated otherwise, we will assume that $X$ is an $\alpha$-stable hyperbolic process in $\mathbb{H}^{d}, 0<\alpha<2$, with the family of Lévy measures $v(x, d y)=v(x, y) \mu(d y)$, which we are going to define now.

First, we introduce an appropriate class of subordinating processes. By $T_{\beta}(t)$ we denote the strictly $\beta$-stable positive standard subordinator with the Laplace transform

$$
\mathbb{E}^{0} e^{-\lambda T_{\beta}(t)}=e^{-t \lambda^{\beta}}, \quad 0<\beta<1 .
$$

Put $\beta=\alpha / 2$ and let $\theta_{\alpha / 2}(t, u), u>0$, denote the density function of $T_{\alpha / 2}(t)$. Let $B(t)$ be a hyperbolic Brownian motion in $\mathbb{H}^{d}$. Then the following process

$$
X(t)=B\left(T_{\alpha / 2}(t)\right)
$$

is the hyperbolic $\alpha$-stable process, under the usual assumption that the processes $T_{\alpha / 2}(t)$ and $B(t)$ are stochastically independent [7,9]. As a subordinated hyperbolic Brownian motion, the process $X(t)$ has the same invariance properties with respect to isometries of $\mathbb{H}^{d}$, as $B(t)$.

By the subordination, the transition density of $X$ with respect to $d \mu$ can be written as

$$
p(t, x, y)=p\left(t, d_{\mathbb{H}^{d}}(x, y)\right)=\int_{0}^{\infty} g^{(d)}\left(u, d_{\mathbb{H}^{d}}(x, y)\right) \theta_{\alpha / 2}(t, u) d u,
$$

and the family of the Lévy measures of the process $X$ as

$$
v(x, d y)=v(x, y) d \mu(y)=c_{\alpha}\left(\int_{0}^{\infty} g^{(d)}\left(u, d_{\mathbb{H}^{d}}(x, y)\right) u^{-1-\alpha / 2} d u\right) d \mu(y) .
$$

Observe that the Lévy density $v(x, y)$ is completely determined by its hyperbolic radial profile, and throughout the paper we write $v$ for the density or its radial profile. Hence $v(x, y)=v\left(d_{\mathbb{H}^{d}}(x, y)\right)$ and it is clear that $v(\eta)$ is a decreasing function of $\eta>0$.

We are interested in the mean exit time of $X$ from $D$, that is, in a function $s_{D}(x)=$ $\mathbb{E}^{x}\left(\tau_{D}\right)$. Here is a connection between the main objects of our study:

$$
s_{D}(x)=\mathbb{E}^{x}\left(\tau_{D}\right)=\int_{\mathbb{H}^{d}} G_{D}(x, y) \mu(d y)=\int_{0}^{\infty} \mathbb{P}^{x}\left(\tau_{D}>t\right) d t .
$$

If $x \in D$, then the $\mathbb{P}^{x}$-distribution of $\left(\tau_{D}, X_{\tau_{D^{-}}}, X_{\tau_{D}}\right.$ ) restricted to $X_{\tau_{D}-} \neq X_{\tau_{D}}$ is given by the following density function (with respect to $d s \times d \mu(u) \times d \mu(z))$ [13],

$$
(0, \infty) \times D \times(\bar{D})^{c} \ni(s, u, z) \mapsto v(u, z) p^{D}(s, x, u) .
$$

For instance, if $x \in D$ and $\mathbb{P}^{x}\left(X_{\tau_{D}-} \in \partial D\right)=0$, then

$$
\mathbb{P}^{x}\left(X_{\tau_{D}} \in d z\right)=\left(\int_{D} G_{D}(x, u) v(u, z) \mu(d u)\right) \mu(d z) \quad \text { on }(\bar{D})^{c} .
$$

Since $B_{d}(t)=B_{d}(0) e^{W(t)-(d-1) t}$, where $W(t)$ is a real Wiener process with variance $2 t$, the transition density of $B_{d}(t)$ (with respect to $\left.\frac{d y_{d}}{y_{d}^{d}}\right)$ is equal to

$$
g\left(t, x_{d}, y_{d}\right)=\frac{1}{\sqrt{4 \pi t}} e^{-t \frac{(d-1)^{2}}{4}}\left(x_{d} y_{d}\right)^{\frac{d-1}{2}} \exp \left[\frac{-\log ^{2}\left(x_{d} / y_{d}\right)}{4 t}\right] \text {. }
$$


To see this, we note that the transition density of $W(t)-(d-1) t$ (with respect to $d y_{d}$ ) equals to

$$
\tilde{g}\left(t, x_{d}, y_{d}\right)=\frac{1}{\sqrt{4 \pi t}} e^{-t \frac{(d-1)^{2}}{4}} e^{-\frac{1}{2}(d-1)\left(y_{d}-x_{d}\right)} \exp \left[\frac{-\left(x_{d}-y_{d}\right)^{2}}{4 t}\right] .
$$

We have $g\left(t, x_{d}, y_{d}\right)=y_{d}^{d} \tilde{g}\left(t, \log x_{d}, \log y_{d}\right) \frac{1}{y_{d}}=y_{d}^{d-1} \tilde{g}\left(t, \log x_{d}, \log y_{d}\right)$ Hence we get the above formula for $g\left(t, x_{d}, y_{d}\right)$.

Using subordination we find that the family of Lévy densities of $X_{d}(t), d \geq 2$, can be written as $L\left(x_{d}, y_{d}\right) \frac{d y_{d}}{y_{d}^{d}}$, with

$$
L\left(x_{d}, y_{d}\right)=\left(x_{d} y_{d}\right)^{(d-1) / 2} v_{\alpha}\left(\log \frac{x_{d}}{y_{d}}\right), x_{d}, y_{d}>0,
$$

where $v_{\alpha}(z)=c_{\alpha, d} \frac{K_{\frac{1+\alpha}{2}}\left(\frac{d-1}{2}|z|\right)}{|z|^{\frac{1+\alpha}{2}}}, z \in \mathbb{R}$, and $K_{\rho}$ is a Macdonald function of index $\rho$. Note that in $\mathbb{H}^{1}$, we have $v_{\alpha}(z)=c_{\alpha} \frac{1}{|z|^{1+\alpha}}$ in the above formula for $L$.

The Lévy measure of $X(t)$ in odd dimension can be calculated explicitly and the resulting formulas involve Macdonald functions. For our purpose it is enough to apply known precise estimates for all $d$. For the reader convenience we provide the proof of this fact.

Lemma 3.1 Let $d \geq 2$. Let $d_{\mathbb{H}^{d}}(x, y)=\eta$. Then

$$
v(x, y)=v(\eta) \approx \frac{1}{\eta^{\alpha}\left(\eta^{1-\alpha / 2}+1\right)} \frac{1}{\mu(B(\eta))},
$$

where $\mu(B(\eta))$ is the volume measure of the hyperbolic ball of radius $\eta$ and $\mu(B(\eta))=$ $\omega_{d-1} \int_{0}^{\eta}(\sinh t)^{d-1} d t \approx(\sinh \eta)^{d-1}(\sinh \eta \wedge 1)$, and $\omega_{d-1}$ is a measure of the unit Euclidean sphere. Moreover, there is a constant $c=c(d, \alpha)$ such that

$$
p(t, \eta) \leq c t v(\eta)
$$

Proof We provide the proof for $d \geq 3$. Similar argument can be carried out for $d=2$, with some obvious modifications, so this case is omitted.

Let $\eta=d_{\mathbb{H}^{d}}(x, y)$. The density of the Lévy measure is given by

$$
v(\eta)=c_{\alpha}\left(\int_{0}^{\infty} g^{(d)}(u, \eta) u^{-1-\alpha / 2} d u\right),
$$

where $g^{(d)}(u, \eta)$ is the transition density of the hyperbolic Brownian motion. We have the following estimate for $g^{(d)}(u, \eta)$ [6]:

$$
g^{(d)}(t, \eta) \approx t^{-d / 2} \exp \left\{-\frac{(d-1)^{2} t}{4}-\frac{(d-1) \eta}{2}-\frac{\eta^{2}}{4 t}\right\}(1+\eta+t)^{(d-3) / 2}(1+\eta) .
$$

Since (here is the point that the assumption $d \geq 3$ matters)

$$
(1+\eta+t)^{(d-3) / 2} \approx(1+\eta)^{(d-3) / 2}+(1+t)^{(d-3) / 2},
$$


we have

$$
\begin{aligned}
g^{(d)}(t, \eta) \approx & t^{-d / 2}(1+t)^{(d-3) / 2}(1+\eta) \exp \left\{-\frac{(d-1)^{2} t}{4}-\frac{(d-1) \eta}{2}-\frac{\eta^{2}}{4 t}\right\} \\
& +t^{-d / 2}(1+\eta)^{(d-1) / 2} \exp \left\{-\frac{(d-1)^{2} t}{4}-\frac{(d-1) \eta}{2}-\frac{\eta^{2}}{4 t}\right\} \\
= & g_{1}(t, \eta)+g_{2}(t, \eta)
\end{aligned}
$$

Then

$$
I_{2}=\int_{0}^{\infty} g_{2}(u, \eta) u^{-1-\alpha / 2} d u=C K_{\frac{d+\alpha}{2}}((d-1) \eta / 2) \eta^{-\frac{d+\alpha}{2}}(1+\eta)^{(d-1) / 2} e^{-\frac{(d-1) \eta}{2}} .
$$

Next, by Eq. 9 with $\eta=0$,

$$
\begin{gathered}
g_{1}(t, \eta) \approx t^{-d / 2}(1+\eta) \exp \left\{-\frac{(d-1)^{2} t}{4}-\frac{(d-1) \eta}{2}-\frac{\eta^{2}}{4 t}\right\} \\
\quad+t^{-3 / 2}(1+\eta) \exp \left\{-\frac{(d-1)^{2} t}{4}-\frac{(d-1) \eta}{2}-\frac{\eta^{2}}{4 t}\right\} \\
=g_{1,1}(t, \eta)+g_{1,2}(t, \eta) \text { and } \\
I_{1}=I_{1,1}+I_{1,2}=\int_{0}^{\infty} g_{1,1}(u, \eta) u^{-1-\alpha / 2} d u+\int_{0}^{\infty} g_{1,2}(u, \eta) u^{-1-\alpha / 2} d u \\
=C_{1} K_{\frac{d+\alpha}{2}}((d-1) \eta / 2) \eta^{-\frac{d+\alpha}{2}}(1+\eta) e^{-\frac{(d-1) \eta}{2}} \\
+C_{2} K_{\frac{3+\alpha}{2}}((d-1) \eta / 2) \eta^{-\frac{3+\alpha}{2}}(1+\eta) e^{-\frac{(d-1) \eta}{2}} .
\end{gathered}
$$

In consequence we have

$$
v(\eta) \approx\left[K_{\frac{d+\alpha}{2}}((d-1) \eta / 2) \eta^{-\frac{d+\alpha}{2}}(1+\eta)^{(d-1) / 2}+K_{\frac{3+\alpha}{2}}((d-1) \eta) \eta^{-\frac{3+\alpha}{2}}(1+\eta)\right] e^{-\frac{(d-1) \eta}{2}} .
$$

Note that $K_{\rho}(z) \approx \frac{1}{\sqrt{z}} e^{-z}, z>1$, hence

$$
v(\eta) \approx \eta^{-\frac{2+\alpha}{2}} e^{-(d-1) \eta}, \eta>1
$$

On the other hand $K_{\rho}(z) \approx \frac{1}{z^{\rho}}, 0<z \leq 1$, and for $0<\eta \leq 1$,

$$
v(\eta) \approx \eta^{-(d+\alpha)} \text {. }
$$

This completes the proof of the estimate of the density of the Lévy measure. Let $\theta_{\alpha / 2}(t, u)$ be the transition density of the $\alpha / 2$-stable subordinator. It is well-known that $\theta_{\alpha / 2}(t, u) \leq c t u^{-1-\alpha / 2}$, which implies the following upper bound

$$
p(t, \eta)=\int_{0}^{\infty} g^{(d)}(u, \eta) \theta(t, u) d u \leq c t \int_{0}^{\infty} g^{(d)}(u, \eta) u^{-1-\alpha / 2} d u=C t v(\eta) .
$$

\section{Mean Exit Time of $X$ from a Halfspace or a Strip}

Throughout the rest of the paper we assume that $d \geq 2$. For $a>0$ define a halfspace $H(a)=\left\{x \in \mathbb{H}^{d}: x_{d}>a\right\}$. In this section we derive a formula for the mean exit time 
from a halfspace $H(a)$. It is clear that mean exit time from $H(a)$ depends only on the last coordinate of the process.

Let $x_{d}>a$ and consider the last coordinate of the hyperbolic stable process starting from $x=\left(x_{1}, \ldots, x_{d}\right)$ in $\mathbb{H}^{d}$. That is $Z(t)=X_{d}(t)=x_{d} \exp \left(Y^{*}(t)\right)$, where $Y^{*}(t)$ is a Brownian motion with drift $W(t)-(d-1) t$, subordinated by an independent $\alpha / 2$-stable subordinator. It is obvious that the first exit time of $X(t)$ from $H(a)$ is the same as the corresponding exit time from the halfline $(a, \infty)$ for the process $X_{d}(t)$. Therefore the problem is one dimensional and reduces to finding the mean exit time from the halfline $\left(\log \frac{a}{x_{d}}, \infty\right)$ of the process $Y^{*}$. Our main observation leading to the solution of the problem relies on finding a connection between the process $Y^{*}$ and the so-called $\alpha$-stable relativistic process.

For fixed $\mu>0$ consider the process $W^{\mu}(t)=W(t)-\mu t, t \geq 0$, where $W(t)$ is a Brownian motion in $\mathbb{R}$ with the characteristic function

$$
\mathbb{E}^{0} e^{i \xi W(t)}=e^{-t|\xi|^{2}}
$$

It is elementary to show that the density of $W^{\mu}(t)$ equals to

$$
g_{t}^{\mu}(x)=e^{-t \frac{\mu^{2}}{4}} e^{-\frac{1}{2} \mu x} g_{t}(x), x \in \mathbb{R},
$$

where $g_{t}(x)$ is the density of $W(t)$.

Now, we subordinate the process $W^{\mu}(t)$ by an independent standard $\alpha / 2$-stable subordinator $T_{\alpha / 2}(t)$, so the resulting process has the following form

$$
Y^{\mu}(t)=W^{\mu}\left(T_{\alpha / 2}(t)\right)
$$

Its transition density is computed as follows. For $x \in \mathbb{R}$ and $t>0$,

$$
\begin{aligned}
p_{t}^{\mu}(x) & =\int_{0}^{\infty} g_{u}^{\mu}(x) \theta_{\alpha / 2}(t, u) d u \\
& =\int_{0}^{\infty} e^{-u \frac{\mu^{2}}{4}} e^{-\frac{1}{2} \mu x} \theta_{\alpha / 2}(t, u) g_{u}(x) d u \\
& =e^{-\frac{1}{2} \mu \cdot x} \int_{0}^{\infty} e^{-u \frac{\mu^{2}}{4}} \theta_{\alpha / 2}(t, u) g_{u}(x) d u .
\end{aligned}
$$

Now, for $m>0$ and $t>0$ define a probability density function on $(0, \infty)$,

$$
\theta_{\alpha / 2}(t, u, m)=e^{m t} \theta_{\alpha / 2}(t, u) e^{-m^{2 / \alpha} u}, \quad u>0 .
$$

Applying Eq. 4 we derive the Laplace transform of $\theta_{\alpha / 2}(t, u, m)$ :

$$
\int_{0}^{\infty} e^{-\lambda u} \theta_{\alpha / 2}(t, u, m) d u=e^{m t} e^{-t\left(\lambda+m^{2 / \alpha}\right)^{\alpha / 2}} .
$$

It is clear that $\theta_{\alpha / 2}(t, u, m)$ defines a density of a subordinator $T_{\alpha / 2}^{m}(t), t>0$. Next we subordinate the Brownian motion $W(t)$ by an independent copy of $T_{\alpha / 2}^{m}(t)$. The resulting process is called the $\alpha$-stable relativistic process with parameter $m$,

$$
R^{m}(t)=W\left(T_{\alpha / 2}^{m}(t)\right), t \geq 0
$$


Its transition density is given by the following formula,

$$
\begin{aligned}
q_{t}^{m}(x) & =\int_{0}^{\infty} \theta_{\alpha / 2}(t, u, m) g_{u}(x) d u \\
& =e^{m t} \int_{0}^{\infty} \theta_{\alpha / 2}(t, u) e^{-m^{2 / \alpha} u} g_{u}(x) d u .
\end{aligned}
$$

Now, take $m$ such that $m^{2 / \alpha}=\frac{\mu^{2}}{4}=\frac{(d-1)^{2}}{4}$. Then, comparing the densities of $R^{m}(t)$ and $Y^{\mu}(t)$, we have

$$
p_{t}^{\mu}(x)=e^{-m^{1 / \alpha} x} e^{-m t} q_{t}^{m}(x) .
$$

From now on we denote $Y(t)=R^{m}(t)$ and observe that $Y^{*}(t)=Y^{\mu}(t)$. The above relationship between $p_{t}^{\mu}$ and $q_{t}^{m}$ implies that the process $Y^{*}(t)$ is absolutely continuous with respect to $Y(t)$ and

$$
\left.\frac{d \mathbb{P}_{*}^{x}}{d \mathbb{P}^{x}}\right|_{\mathcal{F}_{t}}=e^{-m^{1 / \alpha}(Y(t)-x)} e^{-m t},
$$

where $\mathbb{P}_{*}^{x}, \mathbb{P}^{x}$ are distributions of $Y^{*}(t), Y(t)$ respectively and $\mathcal{F}_{t}$ is the $\sigma$-field generated by paths up to time $t$. Let $p_{*}^{D}$ and $p^{D}$ be the corresponding transition densities of the processes $Y^{*}(t)$ and $Y(t)$ killed upon exiting an open set $D \subset \mathbb{R}$.

Lemma 4.1 For every open $D \subset \mathbb{R}$ we have

$$
p_{*}^{D}(t, x, y)=e^{-m^{1 / \alpha}(y-x)} e^{-m t} p^{D}(t, x, y), x, y \in \mathbb{R} .
$$

Moreover, we have the following identity for the Green functions

$$
G_{D}^{*}(x, y)=e^{-m^{1 / \alpha}(y-x)} G_{D}^{m}(x, y), x, y \in \mathbb{R},
$$

where $G_{D}^{m}(x, y)=\int_{0}^{\infty} e^{-m t} p^{D}(t, x, y) d t$ is the m-potential for the killed relativistic process $Y(t)$ with parameter $m$.

Proof Let $f: \mathbb{R} \rightarrow \mathbb{R}$ be a bounded Borel function. Since $\left\{t<\tau_{D}\right\} \subset \mathcal{F}_{t}$ then by Eq. 11 we have

$$
\begin{aligned}
\int_{\mathbb{R}} f(y) p_{*}^{D}(t, x, y) d y & =\mathbb{E}_{*}^{x}\left[f\left(Y_{t}\right), t<\tau_{D}\right]=\mathbb{E}^{x}\left[f\left(Y_{t}\right) e^{-m^{1 / \alpha}(Y(t)-x)} e^{-m t}, t<\tau_{D}\right] \\
& =e^{-m t} \int_{\mathbb{R}} f(y) e^{-m^{1 / \alpha}(y-x)} p^{D}(t, x, y) d y .
\end{aligned}
$$

In particular for $D=(0, \infty)$ and any $m>0$ we have (see [4])

$$
G_{(0, \infty)}^{m}(x, y)=m^{\frac{1-\alpha}{\alpha}} G\left(m^{1 / \alpha} x, m^{1 / \alpha} y\right),
$$

with

$$
G(x, y)=\int_{0}^{x} u(v) u(y-x+v) d v, \quad x<y,
$$


where $u(t)=\frac{1}{\Gamma(\alpha / 2)} t^{\alpha / 2-1} e^{-t}$. Applying Lemma 4.1 we can calculate the Green function of $(0, \infty)$ for $Y_{t}^{*}$ as

$$
\begin{aligned}
G_{(0, \infty)}^{*}(x, y) & =m^{\frac{1-\alpha}{\alpha}} e^{-m^{1 / \alpha}(y-x)} G\left(m^{1 / \alpha} x, m^{1 / \alpha} y\right) \\
& =m^{\frac{1-\alpha}{\alpha}} e^{-m^{1 / \alpha}(y-x)} \int_{0}^{m^{1 / \alpha} x \wedge m^{1 / \alpha} y} u(v) u\left(m^{1 / \alpha}|y-x|+v\right) d v \\
& =m^{\frac{1-\alpha}{\alpha}} e^{-m^{1 / \alpha}(y-x)} \int_{0}^{x \wedge y} u\left(m^{1 / \alpha}(x-v)\right) u\left(m^{1 / \alpha}(y-v)\right) d v \\
& =\frac{1}{\Gamma^{2}(\alpha / 2)} \int_{0}^{x \wedge y}(x-v)^{\alpha / 2-1} e^{-2 m^{1 / \alpha}(y-v)}(y-v)^{\alpha / 2-1} d v \\
& =\int_{0}^{x \wedge y} h^{*}(x-v) h(y-v) d v,
\end{aligned}
$$

where we identify

$$
h^{*}(t)=\frac{1}{\Gamma(\alpha / 2)} t^{\alpha / 2-1}, \quad h(t)=\frac{1}{\Gamma(\alpha / 2)} t^{\alpha / 2-1} e^{-2 m^{1 / \alpha} t} .
$$

Define the lower incomplete Gamma function with index $\alpha / 2$ by the formula $\gamma(\alpha / 2, v)=\int_{0}^{v} y^{\alpha / 2-1} e^{-y} d y, v \geq 0$.

Lemma 4.2 Let $\tau_{(0, R)}^{*}$ be the first exit time of the process $Y^{*}(t)$ from $(0, R), 0<R \leq \infty$. Then for $x>0$ we have

$$
\mathbb{E}^{x} \tau_{(0, \infty)}^{*}=\frac{2}{\alpha m^{1 / 2} \Gamma(\alpha / 2)}\left(\frac{x}{2}\right)^{\alpha / 2}
$$

and

$$
\mathbb{E}^{x} \tau_{(0, R)}^{*} \leq \frac{2}{\alpha \Gamma^{2}(\alpha / 2)} \frac{\gamma\left(\alpha / 2,2 m^{1 / \alpha} R\right)}{m^{1 / 2}}\left(\frac{x}{2}\right)^{\alpha / 2},
$$

where $m^{1 / \alpha}=\frac{d-1}{2}$.

Proof Let $R>0$. Note that

$$
\begin{aligned}
\int_{0}^{R} h(y) d y & =\int_{0}^{R} \frac{1}{\Gamma(\alpha / 2)} y^{\alpha / 2-1} e^{-2 m^{1 / \alpha} y} d y \\
& =\frac{1}{\Gamma(\alpha / 2)} \frac{1}{2^{\alpha / 2} m^{1 / 2}} \int_{0}^{2 m^{1 / \alpha} R} y^{\alpha / 2-1} e^{-y} d y \\
& =\frac{1}{\Gamma(\alpha / 2)} \frac{\gamma\left(\alpha / 2,2 m^{1 / \alpha} R\right)}{2^{\alpha / 2} m^{1 / 2}} .
\end{aligned}
$$


Hence, applying the change of order of integration and Eq. 12 we have

$$
\begin{aligned}
\mathbb{E}^{x} \tau_{(0, \infty)}^{*} & =\int_{0}^{\infty} G^{*}(x, y) d y=\int_{0}^{\infty} d y \int_{0}^{x \wedge y} h^{*}(x-v) h(y-v) d v \\
& =\int_{0}^{x} d v \int_{v}^{\infty} h^{*}(x-v) h(y-v) d y \\
& =\int_{0}^{x} h^{*}(x-v)\left[\int_{v}^{\infty} h(y-v) d y\right] d v \\
& =\int_{0}^{x} h^{*}(x-v)\left[\int_{0}^{\infty} h(y) d y\right] d v \\
& =\frac{1}{\Gamma(\alpha / 2)}\left(\frac{x}{2}\right)^{\alpha / 2} \frac{2}{\alpha m^{1 / 2}} .
\end{aligned}
$$

Similarly,

$$
\begin{aligned}
\mathbb{E}^{x} \tau_{(0, R)}^{*} & \leq \int_{0}^{R} G_{(0, \infty)}^{*}(x, y) d y=\int_{0}^{x} d v \int_{v}^{R} h^{*}(x-v) h(y-v) d y \\
& =\int_{0}^{x} h^{*}(x-v)\left[\int_{v}^{R} h(y-v) d y\right] d v \\
& =\int_{0}^{x} h^{*}(x-v)\left[\int_{0}^{R-v} h(y) d y\right] d v \\
& \leq \int_{0}^{x} h^{*}(x-v)\left[\int_{0}^{R} h(y) d y\right] d v \\
& =\frac{2}{\alpha \Gamma^{2}(\alpha / 2)} \frac{\gamma\left(\alpha / 2,2 m^{1 / \alpha} R\right)}{m^{1 / 2}}\left(\frac{x}{2}\right)^{\alpha / 2} .
\end{aligned}
$$

Recall that $Z=X_{d}$ is the last coordinate of the hyperbolic stable process starting from $x=\left(x_{1}, \ldots, x_{d}\right)$ and $0<a<x_{d}$. Let $\tau=\tau_{(a, \infty)}^{Z}, \sigma=\tau_{\left(-\log \left(x_{d} / a\right), \infty\right)}^{Y^{*}}$ be the corresponding exit times. Noting that they are equal, by Lemma 4.2 we infer

$$
\mathbb{E}^{x_{d}} \tau=\mathbb{E}^{0} \sigma=\mathbb{E}^{\log \left(x_{d} / a\right)} \tau_{(0, \infty)}^{Y^{*}}=\frac{1}{\Gamma(\alpha / 2)} \frac{2^{1-\alpha / 2}}{\alpha m^{1 / 2}}\left(\log \frac{x_{d}}{a}\right)^{\alpha / 2},
$$

where $m=\left(\frac{d-1}{2}\right)^{\alpha}$. Let $S(a, b)=\left\{x \in \mathbb{H}^{d}: a<x_{d}<b\right\}$ be a strip. In a similar way as above, if $\tau=\tau_{(a, b)}^{Z}$ and $\sigma_{(a, b)}=\tau_{\left(-\log \left(x_{d} / a\right),-\log \left(x_{d} / b\right)\right)}^{Y^{*}}, a<x_{d}<b$, then by Lemma 4.2 we obtain

$$
\mathbb{E}^{x} \tau_{S(a, b)} \leq \frac{2}{\alpha \Gamma^{2}(\alpha / 2)} \frac{\gamma\left(\alpha / 2,2 m^{1 / \alpha} \log (b / a)\right)}{m^{1 / 2}}\left(\frac{\log \left(x_{d} / a\right)}{2}\right)^{\alpha / 2}, a<x_{d}<b .
$$

Hence we have the following corollary:

Corollary 4.3 Let $\tau_{a}=\tau_{H(a)}$ be the first exit time of $X$ from $H(a)=\left\{y \in \mathbb{H}^{d}: y_{d}>a\right\}$ and let $x=\left(x_{1}, \ldots, x_{d}\right) \in \mathbb{H}^{d}$ be such that $x_{d}>a$. Then

$$
\mathbb{E}^{x} \tau_{a}=\frac{1}{\Gamma(\alpha / 2)} \frac{2}{\alpha(d-1)^{\alpha / 2}}\left(\log \left(x_{d} / a\right)\right)^{\alpha / 2}=\frac{1}{\Gamma(\alpha / 2)} \frac{2}{\alpha(d-1)^{\alpha / 2}} \delta_{H(a)}^{\alpha / 2}(x),
$$


and

$$
\mathbb{E}^{x} \tau_{S(a, b)} \leq \frac{2}{\alpha \Gamma^{2}(\alpha / 2)} \frac{\gamma\left(\alpha / 2,2 m^{1 / \alpha} \log (b / a)\right)}{m^{1 / 2}}\left(\frac{\log \left(x_{d} / a\right)}{2}\right)^{\alpha / 2},
$$

where $\delta_{D}(x)$ denotes the distance of $x$ from $D^{c}$.

Remark 1 The above observation provides a tool which enables us to develop potential theory for the hyperbolic stable process in a similar way as it was done for subordinate Brownian motions or unimodal isotropic Lévy processes in $\mathbb{R}^{d}[1,15]$. Here the function $g(x)=\mathbb{E}^{x} \tau_{a}=c \delta_{H(a)}^{\alpha / 2}(x)$ is by definition superharmonic in $H(a)$ with respect to the process $X(t)$. That is $g(x) \geq \mathbb{E}^{x} g\left(X\left(\tau_{B(x, r)}\right)\right)$ for any ball $B(x, r) \subset H(a)$. In fact, the action of the Dynkin operator on it gives the value -1 (see Section 5). The knowledge of the shape of a harmonic or superharmonic function should be crucial in finding estimates of the Green functions or heat kernels, at least for smooth domains as in the case of Euclidean subordinate Brownian motions. In this paper we use the above fact to obtain sharp estimates of the mean exit time from a hyperbolic ball of any (hyperbolic) radius.

Remark 2 In the case of the hyperbolic Brownian motion the problem of exiting a strip as above is reduced to the problem of exiting an interval of the one-dimensional standard Brownian motion with drift. The formulas of the Laplace transform of the exit time are well known (see eg. [2]) and the mean exit time can be derived by differentiation of the Laplace transform.

Remark 3 The analogous problem for a ball in $\mathbb{H}^{3}$ was solved in [16], where a formula for the distribution of an exit time was given. Using this distribution the following formula was obtained: for a three-dimensional hyperbolic Brownian motion starting at a point $x$ in $B\left(x_{0}, R\right)$ the mean exit time is given by $\mathbb{E}^{x} \tau_{B\left(x_{0}, R\right)}=\frac{1}{2}(R \operatorname{coth} R-r \operatorname{coth} r)$, where $r=d_{\mathbb{H}^{3}}\left(x, x_{0}\right)$.

The mean exit time from a ball in $\mathbb{H}^{d}$ depends only on the distance of the starting point from the center of the ball. The radial part of the Laplace-Beltrami operator in $\mathbb{H}^{d}$ is equal to $\mathcal{A}=\frac{d^{2}}{d r^{2}}+(d-1) \operatorname{coth} r \frac{d}{d r}$ (compare e.g. [11]). As it was proved in [8], the problem of finding $s_{R}(r)=\mathbb{E}^{x} \tau_{B\left(x_{0}, R\right)}$, where $r=d_{\mathbb{H} d}\left(x, x_{0}\right)$, is equivalent to solving the following boundary value problem:

$$
y^{\prime \prime}(r)+(d-1) \operatorname{coth} r y^{\prime}(r)=-1, \quad y(R)=0, \quad y(0) \text { is finite. }
$$

In particular, the solution for $0<r<R$ is equal to

$$
\begin{array}{ll}
s_{R}(r)=\ln (1+\cosh R)-\ln (1+\cosh r) & \text { for } d=2, \\
s_{R}(r)=\frac{1}{2}(R \operatorname{coth} R-r \operatorname{coth} r) & \text { for } d=3, \\
s_{R}(r)=\frac{\ln ((\sinh R) / \tanh (R / 2))-1 /(1+\cosh R)}{3}-\frac{\ln ((\sinh r) / \tanh (r / 2))-1 /(1+\cosh r)}{3} & \text { for } d=4, \\
s_{R}(r)=\frac{1+R \sinh (2 R)-R \operatorname{coth} R}{8 \sinh ^{2} R}-\frac{1+r \sinh (2 r)-r \operatorname{coth} r}{8 \sinh ^{2} r} & \text { for } d=5 .
\end{array}
$$

In principle it is possible to solve the above boundary value problem for any $d \geq 2$ and the solution is an elementary function but we were not able to give one general formula for all dimensions.

Remark 4 Below we show how to obtain sharp estimates of the mean exit time from a ball for a hyperbolic Brownian motion using the argument based on Dynkin's lemma. For $r \geq 0$, 
let $g(r)=g_{R}(r)=r^{2}$ if $R \leq 1$ or $g(r)=g_{R}(r)=r^{2} I_{0 \leq r<1}+(2 r-1) I_{r \geq 1}$ if $R \geq 1$. By elementary calculations

$$
2(d-1) \leq \mathcal{A} g(r) \leq 5(d-1) .
$$

Let $Z(t)$ be the diffusion generated by $\mathcal{A}$. Since $(d-1) \operatorname{coth} r \sim \frac{d-1}{r}, r \rightarrow 0, d \geq 2$, the diffusion never hits 0 if started from $r>0$. Hence by the Dynkin lemma

$$
\mathbb{E}^{r} \int_{0}^{\tau_{(0, R)}} \mathcal{A} g(Z(t)) d t=\mathbb{E}^{r} g\left(Z\left(\tau_{(0, R)}\right)\right)-g(r)=g(R)-g(r), \quad 0<r<R .
$$

By Eq. 13,

$$
(g(R)-g(r)) \frac{1}{5(d-1)} \leq \mathbb{E}^{r} \tau_{(0, R)} \leq \frac{1}{2(d-1)}(g(R)-g(r)) .
$$

Again, by elementary calculations we show that $g(R)-g(r) \approx \frac{R-r}{d} \tanh R, 0<r<R$.

\section{Mean Exit Time of $X$ from a Ball}

Using the fact that every ball of a given radius $R$ is isometric (in hyperbolic geometry) to a ball of the same radius $R$ but centred at $x_{0}=(0, . ., 0,1)$, we consider only the ball $B\left(x_{0}, R\right)$. Using the above result for a strip, we can obtain an upper estimate of the mean exit time from the hyperbolic ball of radius $R$. Let $x \in B\left(x_{0}, R\right)$. There is an isometry $I$ such that $I\left(x_{0}\right)=x_{0}$ and $I(x)=\left(0, \ldots, 0, x_{d}^{*}\right)=x^{*}$, where $x_{d}^{*}=e^{-R} e^{\delta_{B\left(x_{0}, R\right)}(x)}$. Since $I$ is an isometry, the processes $X$ and $I(X)$ have the same distribution and we have

$$
\mathbb{E}^{x} \tau_{B\left(x_{0}, R\right)}=\mathbb{E}^{x^{*}} \tau_{B\left(x_{0}, R\right)} .
$$

It is evident that $\delta_{B\left(x_{0}, R\right)}(x)=\delta_{B\left(x_{0}, R\right)}\left(x^{*}\right)$. Inserting the ball $B\left(x_{0}, R\right)$, which is an Euclidean ball with the center $x_{0}^{*}=(0, . ., 0, \cosh R)$, in the strip $S\left(e^{-R}, e^{R}\right)$ tangent to the ball, by Corollary 4.3 we have

$\mathbb{E}^{x} \tau_{B\left(x_{0}, R\right)}=\mathbb{E}^{x^{*}} \tau_{B\left(x_{0}, R\right)} \leq \mathbb{E}^{x^{*}} \tau_{S\left(e^{-R}, e^{R}\right)} \leq \frac{2^{1-\alpha / 2}}{\alpha \Gamma^{2}(\alpha / 2)} \frac{\gamma\left(\alpha / 2,4 m^{1 / \alpha} R\right)}{m^{1 / 2}}\left(\delta_{B\left(x_{0}, R\right)}(x)\right)^{\alpha / 2}$.

Because $\gamma\left(\alpha / 2,4 m^{1 / \alpha} R\right) \leq C(\tanh R)^{\alpha / 2}$, this can be rewritten as

$$
\mathbb{E}^{x} \tau_{B\left(x_{0}, R\right)} \leq C\left(\delta_{B\left(x_{0}, R\right)}(x) \tanh R\right)^{\alpha / 2},
$$

where the constant $C=C(\alpha, d)$.

Our aim is to show that the right hand side of the above inequality provides the right order of the lower bound of the mean exit time. To this end we first establish the lower bound if $x \in B\left(x_{0}, R / 2\right)$. It is well known that $\mu(d y)$ (the volume measure in $\mathbb{H}^{d}$ ) can be written as

$$
\mu(d y)=(\sinh \rho)^{d-1} d \rho \Omega_{d-1}(d s),
$$

where $\Omega_{d-1}(d s)$ is the usual surface measure on the unit sphere in $\mathbb{R}^{d}$ and $\rho=d_{\mathbb{H}^{d}}\left(x_{0}, y\right)$. It follows that for any radial function $f(y)=F(\rho)$ we have

$$
\int_{\mathbb{H}^{d}} f(y) \mu(d y)=\omega_{d-1} \int_{0}^{\infty} F(\rho)(\sinh \rho)^{d-1} d \rho,
$$

where $\omega_{d-1}$ is a measure of the unit Euclidean sphere.

Lemma 5.1 There is $c=c(\alpha, d)$ such that for all $r, t>0$,

$$
\mathbb{P}^{x}\left(d_{\mathbb{H}^{d}}(X(t), x) \geq r\right) \leq c \frac{t}{r^{\alpha} \wedge r^{\alpha / 2}} .
$$


Proof By virtue of Lemma 3.1, we have the following estimate of the $\alpha$-stable heat kernel (with respect to $\mu(d y)$ )

$$
p(t, x, y) \leq C \frac{t}{\rho^{\alpha}\left(\rho^{1-\alpha / 2}+1\right)} \frac{1}{\mu(B(\rho))}, \quad \rho=d_{\mathbb{H}^{d}}(x, y),
$$

where $\mu(B(\rho)) \approx(\sinh \rho)^{d-1}(1 \wedge \sinh \rho)$. Hence for $r>0$ we have

$$
\mathbb{P}^{x}\left(d_{\mathbb{H}^{d}}(X(t), x) \geq r\right) \leq c t \int_{r}^{\infty} \frac{1}{\rho^{\alpha}\left(\rho^{1-\alpha / 2}+1\right)(1 \wedge \rho)} d \rho \leq c \frac{t}{r^{\alpha / 2} \wedge r^{\alpha}} .
$$

Applying a standard trick based on the strong Markov property and Lemma 5.1, we obtain the following estimate

$$
\mathbb{P}^{x}\left(\tau_{B\left(x_{0}, R\right)} \leq t\right) \leq C \frac{t}{R^{\alpha / 2} \wedge R^{\alpha}}, \quad d_{\mathbb{H}^{d}}\left(x_{0}, x\right) \leq 3 R / 4,
$$

where $C=C(\alpha, d)$. Indeed, for $d_{\mathbb{H} d}\left(x, x_{0}\right) \leq 3 R / 4$ we have

$$
\begin{aligned}
& \mathbb{P}^{x}\left(\tau_{B\left(x_{0}, R\right)} \leq t\right) \leq \mathbb{P}^{x}\left(\tau_{B\left(x_{0}, R\right)} \leq t ; d_{\mathbb{H}^{d}}(X(t), x) \leq R / 8\right)+\mathbb{P}^{x}\left(d_{\mathbb{H}^{d}}(X(t), x) \geq R / 8\right) \\
& \leq \mathbb{P}^{x}\left(\tau_{B\left(x_{0}, R\right)} \leq t ; d_{\mathbb{H} d}\left(X(t), X\left(\tau_{B\left(x_{0}, R\right)}\right)\right)\right. \\
& \geq R / 8)+\mathbb{P}^{x}\left(d_{\mathbb{H} d}(X(t), x) \geq R / 8\right) \\
& =\mathbb{E}^{X}\left(\tau_{B\left(x_{0}, R\right)} \leq t ; P^{X\left(\tau_{B\left(x_{0}, R\right)}\right)}\left(d_{\mathbb{H} d}\left(X\left(t-\tau_{B\left(x_{0}, R\right)}\right), X\left(\tau_{B\left(x_{0}, R\right)}\right)\right) \geq R / 8\right)\right. \\
& +\mathbb{P}^{x}\left(d_{\mathbb{H} d}(X(t), x) \geq R / 8\right) \\
& \leq 2 \sup \sup _{s \leq t} \mathbb{P}^{y}\left(d_{\mathbb{H}^{d}}(X(s), y) \geq R / 8\right) \\
& \leq C \frac{t}{R^{\alpha} \wedge R^{\alpha / 2}}
\end{aligned}
$$

In consequence there is $c=c(\alpha, d)$ such that

$$
\mathbb{P}^{x}\left(\tau_{B\left(x_{0}, R\right)} \geq c\left(R^{\alpha / 2} \wedge R^{\alpha}\right)\right) \geq 1 / 2, d_{\mathbb{H}^{d}}\left(x, x_{0}\right) \leq 3 R / 4 .
$$

Let $t_{0}=c\left(R^{\alpha / 2} \wedge R^{\alpha}\right)$. Applying Chebyshev's inequality, we get

$$
\mathbb{E}^{x} \tau_{B\left(x_{0}, R\right)} \geq t_{0} \mathbb{P}^{x}\left(\tau_{B\left(x_{0}, R\right)} \geq t_{0}\right) \geq t_{0} / 2=(c / 2)\left(R^{\alpha / 2} \wedge R^{\alpha}\right) \approx(R \tanh R)^{\alpha / 2} .
$$

As a corollary, we have the following estimate, which agrees with Eq. 14 for $d_{\mathbb{H}^{d}}\left(x, x_{0}\right) \leq 3 R / 4$ :

$$
\mathbb{E}^{x} \tau_{B\left(x_{0}, R\right)} \approx(R \tanh R)^{\alpha / 2}, d_{\mathbb{H}^{d}}\left(x, x_{0}\right) \leq 3 R / 4 .
$$

Here we mention that the above estimate for large radii, say $R>1$, was obtained in [18]. Our aim is to prove the following two-sided estimate of the mean exit time which is sharp for all starting points.

Theorem 5.2 For all $x \in B\left(x_{0}, R\right)$,

$$
\mathbb{E}^{x} \tau_{B}\left(x_{0}, R\right) \approx\left(\delta_{B\left(x_{0}, R\right)}(x) \tanh R\right)^{\alpha / 2}, x \in B\left(x_{0}, R\right) .
$$

To complete the proof of this theorem, we need some preparatory results. For $f: \mathbb{H}^{d} \rightarrow$ $\mathbb{R}, t>0$ and $x \in \mathbb{H}^{d}$ we consider the (approximating) Dynkin operator,

$$
\mathcal{A}_{t} f(x)=\frac{\mathbb{E}^{x} f\left(X\left(\tau_{B(x, t)}\right)\right)-f(x)}{\mathbb{E}^{x} \tau_{B(x, t)}},
$$


whenever $\mathbb{E}^{x} f\left(X\left(\tau_{B(x, t)}\right)\right)$ is well defined. For instance, if $s_{D}(x)=\mathbb{E}^{x} \tau_{D}$ and $0<t \leq$ $\delta_{D}(x)$, then by the strong Markov property, $s_{D}(x)=s_{B(x, t)}(x)+\mathbb{E}^{x} s_{D}\left(X_{\tau_{B(x, t)}}\right)$, and hence

$$
\mathcal{A}_{t} s_{D}(x)=-1 \text {. }
$$

Suppose now that $I$ is an isometry of $\mathbb{H}^{d}$ and $f(x)$ is such function that $f(x)=f(I x)$ for $x \in \mathbb{H}^{d}$. For instance, let $f$ depend only on the distance $d_{\mathbb{H}^{d}}\left(x, x_{0}\right)$ and $x_{0}$ is a fixed point of $I$. Then, by the invariance of $X$ with respect to $I$, it is clear that for such $f$ and $I$

$$
\mathbb{E}^{x} f(X(t))=\mathbb{E}^{I x} f(I(X(t)))=\mathbb{E}^{I x} f(X(t)) .
$$

It follows that for such functions from the domain of the usual generator $\mathcal{A}$ of $X(t)$ we have

$$
\mathcal{A} f(x)=\mathcal{A} f(I x)
$$

The same is true for the Dynkin type generator $\mathcal{A}_{t}$ since $\mathbb{P}^{x}$ distribution of $X\left(\tau_{B(x, t)}\right)$ is the same as $\mathbb{P}^{I x}$ distribution of $X\left(\tau_{B(I x, t)}\right)$.

We also observe the following minimum principle: if $x$ is a point in $\mathbb{H}^{d}$ and $f(x)=$ $\inf _{y \in \mathbb{H}^{d}} f(y)$, then $\mathcal{A}_{t} f(x) \geq 0$ for every $t>0$. This implies the following corollary.

Corollary 5.3 If $\mathcal{A}_{t} f(x)<0$ for some $t>0$, then $f(x)>\inf _{y \in \mathbb{H}^{d}} f(y)$.

Lemma 5.4 Let $D \subset \mathbb{H}^{d}$ be bounded. If $f: \mathbb{H}^{d} \mapsto \mathbb{R}$ is continuous, $f \geq 0$ on $D^{c}$ and for every $x \in D$ there is $t>0$ such that $\mathcal{A}_{t} f(x)<0$, then $f \geq 0$ on $\mathbb{H}^{d}$.

Proof Suppose that inf ${ }_{y \in \mathbb{H}^{d}} f(y)<0$. Then by continuity and the fact $f$ is non-negative on $D^{c}$, the infimum must be attained at $x_{0} \in D$. This is a contradiction to Corollary 5.3.

Consider $D=B\left(x_{0}, R\right)$, where $x_{0}=\left(0, \ldots, 0, x_{d}\right)$, and observe that $D$ is tangent to $H(a)$, where $a=e^{-R}$. Let $f(y)=\delta_{B\left(x_{0}, R\right)}^{\alpha / 2}(y)$. Our main technical result is the estimate of $\mathcal{A}_{t} f(x)$ for $x$ close to the boundary of $D$. Since the function $f(x)$ is invariant for hyperbolic rotations around $x_{0}$, we may choose the point $x$ conveniently, since for any other $w$ with the same distance to the boundary of $D$ there holds $\mathcal{A}_{t} f(x)=\mathcal{A}_{t} f(w)$. Therefore we take $x=\left(0, \ldots, 0, x_{d}\right) \in D$ such that $e^{-R}<x_{d} \leq e^{-3 R / 4}$, that is $\delta_{B\left(x_{0}, R\right)}(x) \leq R / 4$. Let $h(y)=\left(\delta_{H(a)}(y)\right)^{\alpha / 2}=\left(R+\log y_{d}\right)^{\alpha / 2}, y \in H(a)$. The function $h$ will play important role in estimating the Dynkin generator applied to $f$.

Proposition 5.5 Let $R \leq 1$. Let $f(x)=\delta_{B\left(x_{0}, R\right)}^{\alpha / 2}(x)$. There is $C=C(\alpha, d)$ such that for sufficiently small $t$ and $x \in B\left(x_{0}, R\right)$ such that $\delta_{B\left(x_{0}, R\right)}(x) \leq R / 4$ we have

$$
0 \leq-\mathcal{A}_{t} f(x) \leq C \frac{1}{R^{\alpha / 2}}
$$

The above estimate is a key tool to obtain the lower bound of the mean exit time from a ball. Its proof, quite long and technical, requires a number of preliminary results and is postponed to the last section. Now we prove continuity of the mean exit time from a ball regarded as a function of the starting point of the process. The proof follows the arguments of Lemma 2.9 of [1].

Proposition 5.6 For every $R>0$ the function $s(x)=\mathbb{E}^{x} \tau_{B\left(x_{0}, R\right)}$ is continuous in $\mathbb{H}^{d}$. 
Proof First, if $x \notin B\left(x_{0}, R\right)$, then $s(x)=0$. If $H(a)=\left\{x \in \mathbb{H}^{d}: x_{d}>a\right\}$ is such a halfspace containing $B\left(x_{0}, r\right)$ that the boundary of $H(a)$ is tangent to the ball, then evidently $\tau_{H(a)} \geq \tau_{B\left(x_{0}, R\right)}$. By Corollary 4.3, if $x$ approaches boundary of $H(a)$ then $\mathbb{E}^{x} \tau_{H(a)}$ tends to zero, hence the same is true for $\mathbb{E}^{x} \tau_{B\left(x_{0}, R\right)}$, so that $s(x)$ is continuous on $B^{c}\left(x_{0}, R\right)$. Now let $x \in B\left(x_{0}, R\right)$ and let $z$ tend to $x$. By the invariance of the process $X$ with respect to the hyperbolic isometries, the function $s(x)=\mathbb{E}^{x} \tau_{B\left(x_{0}, R\right)}$ is constant on every sphere $S\left(x_{0}, r\right), 0<r<R$. We also can and do assume that $x_{0}=(0, \ldots, 0,1)$ and points $x, z$ are of the following form $x=\left(0, \ldots, 0, x_{d}\right)$ and $z=\left(0, \ldots, 0, z_{d}\right)$. Denote this ball by $B$. Assume also that $x_{d}>z_{d}$ and consider a ball $B^{\prime}=B\left(\left(0, \ldots, 0, \frac{z_{d}}{x_{d}}\right), R\right)$. If $x_{d}<$ $z_{d}$ then we consider a ball $B^{\prime}=B\left(\left(0, \ldots, 0, \frac{x_{d}}{z_{d}}\right), R\right)$. Observe that the distance from $x$ to the complement of $B$ is the same as the distance of $z$ to the of boundary $B^{\prime}$, hence $\mathbb{E}^{x}\left(\tau_{B}\right)=\mathbb{E}^{z}\left(\tau_{B^{\prime}}\right)$. Now let $U=B \cap B^{\prime}$ and $W=B \backslash U$. By the strong Markov property $s_{B}(x)=s_{U}(x)+\int_{W} s_{D}(y) \omega_{U}^{x}(d y)$, where $\omega_{U}^{x}(d y)$ is a harmonic measure of $U$ and $X$ starts from $x$. If $z$ tends to $x$ then for $y \in W$ there holds $\delta_{B}(y) \leq d_{\mathbb{H}^{d}}(x, z) \rightarrow 0$. Fix $y \in W$. Using a hyperbolic rotation we can rotate the ball $B^{\prime}$ around its center in such a way that $y \in W$ is transformed into a point $w$ with first $d-1$ coordinates equal to zero. Then, using inclusion $B^{\prime}$ in a half-space $H(a)$ tangent to the boundary of $B^{\prime}$, we get $\tau_{B^{\prime}} \leq \tau_{H(a)}$ and $\mathbb{E}^{w}\left(\tau_{H(a)}\right)$ goes to zero, as $d_{\mathbb{H} d}(x, y)$ tends to zero. But $s_{B}(x)=s_{B^{\prime}}(z) \geq s_{U}(x)$ hence

$$
s_{B}(x) \geq s_{B^{\prime}}(z)-\int_{R} s_{D}(y) \omega_{U}^{x}(d y) \rightarrow s_{B}(z), \text { as } d_{\mathbb{H} d}(x, z) \rightarrow 0 .
$$

Changing the roles of $x$ and $z$, we get the continuity of $s(x)=s_{B}(x)$ for all $x \in \mathbb{H}^{d}$.

Theorem 5.7 Let $0<R \leq 1$. There is a constant $C=C(\alpha, d)$ such that

$$
\mathbb{E}^{x} \tau_{B\left(x_{0}, R\right)} \geq C\left(\delta_{B\left(x_{0}, R\right)}(x)\right)^{\alpha / 2} R^{\alpha / 2}, \quad x \in \mathbb{H}^{d} .
$$

Proof Let $B_{R}=B\left(x_{0}, R\right)$. Recall that $\delta_{B_{R}}(x)$ denotes the distance from $x$ to $B_{R}^{c}$, hence Eq. 18 holds on $\bar{B}_{R}^{c}$. Denote $s(x)=\mathbb{E}^{x} \tau_{B_{R}}$ and $f(x)=\left(\delta_{B_{R}}(x)\right)^{\alpha / 2}, x \in \mathbb{H}^{d}$. Due to Eq. 16, $s(x) \approx R^{\alpha}$ on $\overline{B_{3 R / 4}}$, hence there is $C=C(d, \alpha)$ so large that

$$
C s(x)-R^{\alpha / 2} f(x) \geq 0 \quad \text { if } \quad \delta_{B_{R}}(x) \geq R / 4 .
$$

Let $0<\delta_{B_{R}}(x)<R / 4$. If $t>0$ is small, then by Proposition 5.5 we have $\left|\mathcal{A}_{t} f(x)\right| \leq$ $c / R^{\alpha / 2}$, and by Eq. 17 we obtain

$$
\mathcal{A}_{t}\left[(c+1) s-R^{\alpha / 2} f\right](x)=-(c+1)-R^{\alpha / 2} \mathcal{A}_{t} f(x) \leq-1 .
$$

Let $c^{*}=C \vee(c+1)$. By Proposition 5.6, the function $g(x)=c^{*} s(x)-R^{\alpha / 2} f(x)$ is continuous. By Lemma 5.4, with $D=B_{R} \backslash \overline{B_{3 R / 4}}$ we have $g \geq 0$ everywhere.

We are now ready to provide the proof of Theorem 5.2.

Proof By Eq. 14 and Theorem 5.7 it is enough to deal with the lower bound in the case $R>1$. If $R>1$ and $\delta_{B\left(x_{0}, R\right)}(x)<1$ then we can find $x^{*} \in B\left(x_{0}, R\right)$ such that $B\left(x^{*}, 1\right) \subset$ $B\left(x_{0}, R\right)$ and $\delta_{B\left(x_{0}, R\right)}(x)=\delta_{B\left(x^{*}, 1\right)}(x)$. Hence from Theorem 5.7 we have

$$
\mathbb{E}^{x} \tau_{B\left(x_{0}, R\right)} \geq \mathbb{E}^{x} \tau_{B\left(x^{*}, 1\right)} \geq C\left(\delta_{B\left(x^{*}, 1\right)}(x)\right)^{\alpha / 2}=C\left(\delta_{B\left(x_{0}, R\right)}(x)\right)^{\alpha / 2} .
$$


If $R>1$ and $\delta_{B\left(x_{0}, R\right)}(x)>1$ we note that by Eq. 16,

$$
\mathbb{E}^{x} \tau_{B\left(x_{0}, R\right)} \geq \mathbb{E}^{x} \tau_{B\left(x, \delta_{B\left(x_{0}, R\right)}(x)\right)} \geq C\left(\delta_{B\left(x_{0}, R\right)}(x)\right)^{\alpha / 2} .
$$

\section{Estimates of the Dynkin Generator in $d$-Dimensional Case}

In this section we prove Proposition 5.5. The proof requires a number of preliminary lemmas. For $y \in \mathbb{H}^{d}, d \geq 2$, we write $y=\left(\tilde{y}, y_{d}\right)$, where $\tilde{y}=\left(y_{1}, \ldots, y_{d-1}\right)$.

Lemma 6.1 Let $R \leq 1$. Let $y \in B\left(A^{*}, R / 2\right)$ such that $y_{d}<e^{-R / 2}$, where $A^{*}=\left(\tilde{0}, e^{-R / 2}\right)$. Let $H=H\left(e^{-R}\right)$ and $D=B\left(x_{0}, R\right)$. Then

$$
3 \delta_{D}(y) \geq \delta_{H}(y) .
$$

Proof Note that if we keep $y_{d}$ fixed then $\delta_{D}(y)$ is the smallest on the sphere of $B\left(A^{*}, R / 2\right)$. Hence we may assume that $d_{\mathbb{H} d}\left(y, A^{*}\right)=R / 2$. We have

$$
\cosh (R / 2)=1+\frac{\left|y-A^{*}\right|^{2}}{2 y_{d} e^{-R / 2}}=1+\frac{\left|y_{d}-e^{-R / 2}\right|^{2}}{2 y_{d}} e^{R / 2}+\frac{|\tilde{y}|^{2}}{2 y_{d}} e^{R / 2}
$$

and

$$
\cosh d_{\mathbb{H}^{d}}\left(x_{0}, y\right)=1+\frac{\left|y_{d}-1\right|^{2}}{2 y_{d}}+\frac{|\tilde{y}|^{2}}{2 y_{d}},
$$

which implies that

$$
\cosh d_{\mathbb{H}^{d}}\left(x_{0}, y\right)=\frac{1+e^{-R}}{2}+\frac{1-e^{-R}}{2 y_{d}} .
$$

By convexity of the function $\cosh x$ we have

$$
\begin{aligned}
3 \delta_{D}(y) & =3\left(R-d_{\mathbb{H} d}\left(x_{0}, y\right)\right) \geq 3 \frac{\cosh R-\cosh d_{\mathbb{H}^{d}}\left(x_{0}, y\right)}{\sinh R} \\
& =3 \frac{\cosh R-\frac{1+e^{-R}}{2}-\frac{1-e^{-R}}{2 y_{d}}}{\sinh R} \\
& =3 \frac{\frac{e^{R}-1}{2}}{\sinh R}\left(1-\frac{1-e^{-R}}{y_{d}\left(e^{R}-1\right)}\right) \geq \frac{3}{2}\left(1-\frac{1-e^{-R}}{y_{d}\left(e^{R}-1\right)}\right),
\end{aligned}
$$

because $e^{R}-1 \geq \sinh R$ for all $R$. Since $\delta_{H}(y)=\left(R+\log y_{d}\right)$, in order to prove the lemma, we must show that for $0<R<1$ and $e^{-R}<y_{d}<e^{-R / 2}$,

$$
\frac{3}{2}\left(1-\frac{1-e^{-R}}{y_{d}\left(e^{R}-1\right)}\right) \geq\left(R+\log y_{d}\right) .
$$

But the function $w\left(y_{d}\right)=\frac{3}{2}\left(1-\frac{1-e^{-R}}{y_{d}\left(e^{R}-1\right)}\right)-\left(R+\log y_{d}\right)$ is increasing for $e^{-R}<y_{d}<$ $e^{-R / 2}$, and $w\left(e^{-R}\right)=0$, so that the proof is completed.

Lemma 6.2 Let $_{\mathbb{H}^{d}}(x, y) \leq 1$ and $x_{d}, y_{d} \leq 1$. Then $d_{\mathbb{H}^{d}}(x, y) \geq|x-y| / 2$. 
Proof Observe that

$$
2 \geq \cosh 1 \geq \cosh d_{\mathbb{H}^{d}}(x, y)=1+\frac{|x-y|^{2}}{2 x_{d} y_{d}} \geq 1+\frac{|x-y|^{2}}{2} .
$$

Applying the inequality $1+t \geq e^{t / 2}, 0 \leq t \leq 1$, with $t=\frac{|x-y|^{2}}{2}$, we arrive at

$$
e^{d_{\mathbb{H}}(x, y)^{2}} \geq \cosh d_{\mathbb{H} d}(x, y) \geq 1+\frac{|x-y|^{2}}{2} \geq e^{\frac{|x-y|^{2}}{4}} .
$$

Lemma 6.3 Let $R<1$. If $y=\left(\tilde{y}, y_{d}\right) \in B\left(x_{0}, R\right)$, with $y_{d}<e^{-R / 2}$, then

$$
\delta_{H}(y)-\delta_{B\left(x_{0}, R\right)}(y) \leq 3 \frac{|\tilde{y}|^{2}}{R} .
$$

Proof Let $A=\left(\tilde{0}, e^{-R}\right)$. First note that $\delta_{H}(y)=d_{\mathbb{H}^{d}}\left(\left(\tilde{0}, y_{d}\right), A\right)=R+\log y_{d}$ and $\delta_{B\left(x_{0}, R\right)}(y)=R-d_{\mathbb{H}^{d}}\left(y, x_{0}\right)$, hence

$$
\delta_{H}(y)-\delta_{B\left(x_{0}, R\right)}(y)=d_{\mathbb{H}^{d}}\left(y, x_{0}\right)-d_{\mathbb{H}^{d}}\left(\left(\tilde{0}, y_{d}\right), x_{0}\right)
$$

Using convexity of the function $\cosh x$ and applying Eq. 1 we obtain

$$
\begin{aligned}
\delta_{H}(y)-\delta_{B\left(x_{0}, R\right)}(y) & =d_{\mathbb{H}^{d}}\left(y, x_{0}\right)-d_{\mathbb{H}^{d}}\left(\left(\tilde{0}, y_{d}\right), x_{0}\right) \\
& \leq \frac{\cosh d_{\mathbb{H}^{d}}\left(y, x_{0}\right)-\cosh d_{\mathbb{H} d}\left(\left(\tilde{0}, y_{d}\right), x_{0}\right)}{\sinh d_{\mathbb{H}^{d}}\left(y, x_{0}\right)} \\
& =\frac{\left|y-x_{0}\right|^{2}-\left|\left(\tilde{0}, y_{d}\right)-x_{0}\right|^{2}}{2 y_{d} \sinh d_{\mathbb{H}^{d}}\left(y, x_{0}\right)} \\
& \leq \frac{|\tilde{y}|^{2}}{2 e^{-R} \sinh (R / 2)} \\
& \leq 3 \frac{|\tilde{y}|^{2}}{R} .
\end{aligned}
$$

Lemma 6.4 Let $R>0$ and $H=H\left(e^{-R}\right)$. Let $h(y)=\left(\delta_{H}(y)\right)^{\alpha / 2}=\left(R+\log y_{d}\right)^{\alpha / 2}, y \in$ $\mathbb{H}^{d}$. There is $C=C(\alpha, d)$ such that for any $w \in \mathbb{H}^{d}, w_{d}<e^{-R / 2}$,

$$
\begin{aligned}
\int_{\left\{y_{d}>1\right\}} h(y) v(w, y) \mu(d y) & =\int_{\left\{y_{d}>1\right\}}\left(\log y_{d}+R\right)^{\alpha / 2} v(w, y) \mu(d y) \\
& \leq C w_{d}^{(d-1) / 2}\left(\frac{1}{R^{\alpha / 2}} \vee 1\right) .
\end{aligned}
$$


Proof Applying Eq. 8, which provides the form of the Lévy density of $X_{d}$, we get

$$
\begin{aligned}
\int_{\left\{y_{d}>1\right\}} h(y) v(w, y) \mu(d y) & =\int_{\left\{y_{d}>1\right\}}\left(\log y_{d}+R\right)^{\alpha / 2} v(w, y) \mu(d y) \\
& =\int_{1}^{\infty}\left(\log y_{d}+R\right)^{\alpha / 2} L\left(w_{d}, y_{d}\right) \frac{d y_{d}}{y_{d}^{d}} \\
& =\int_{1}^{\infty}\left(\log y_{d}+R\right)^{\alpha / 2}\left(w_{d} y_{d}\right)^{(d-1) / 2} v_{\alpha}\left(\log w_{d}-\log y_{d}\right) \frac{d y_{d}}{y_{d}^{d}} \\
& =w_{d}^{(d-1) / 2} \int_{0}^{\infty}(u+R)^{\alpha / 2} e^{-(d-1) u / 2} v_{\alpha}\left(\log w_{d}-u\right) d u \\
& \leq w_{d}^{(d-1) / 2} \int_{0}^{\infty}(u+R)^{\alpha / 2} e^{-(d-1) u / 2} v_{\alpha}(u+R / 2) d u \\
& \leq 2^{\alpha / 2} w_{d}^{(d-1) / 2} \int_{R / 2}^{\infty} z^{\alpha / 2} v_{\alpha}(z) d z \\
& \leq C w_{d}^{(d-1) / 2}\left(\frac{1}{R^{\alpha / 2}} \vee 1\right) .
\end{aligned}
$$

The last estimate follows from the estimate $v_{\alpha}(z) \approx \frac{1+z^{\alpha / 2}}{\left(1+z^{1 / 2}\right) z^{1+\alpha}} e^{-z}, z>0$, which is a consequence of the asymptotic behaviour of Macdonald functions.

Now we are ready to prove Proposition 5.5.

Proof Let $D=B\left(x_{0}, R\right)$. Note that $D$ is tangent to $H=H\left(e^{-R}\right)$. For $y \in \mathbb{H}^{d}$ let $h(y)=$ $\left(\delta_{H}(y)\right)^{\alpha / 2}$ and $f(y)=\left(\delta_{D}(y)\right)^{\alpha / 2}$. Since the function $f(y)$ is invariant for hyperbolic rotations around $x_{0}$, we may choose the point $x$ conveniently since for any other $w \in D$ with the same distance to the boundary of $D$, we have $\mathcal{A}_{t} f(x)=\mathcal{A}_{t} f(w)$. Therefore we take $x=\left(\tilde{0}, x_{d}\right) \in D$ such that $e^{-R}<x_{d} \leq e^{-3 R / 4}$, that is, $\delta_{B\left(x_{0}, R\right)}(x) \leq R / 4$. We let $R(y)=h(y)-f(y) \geq 0, y \in \mathbb{H}^{d}$, and choose $0<t \leq\left(R+\log x_{d}\right) / 2 \leq R / 8$. Since $B(x, t) \subset H$, Corollary 4.3 and Eq. 17 imply $\mathcal{A}_{t} h(x)=-\frac{\alpha}{2} \Gamma(\alpha / 2)(d-1)^{\alpha / 2}<0$, so that we obtain

$$
-\mathcal{A}_{t} f(x)=\mathcal{A}_{t} R(x)+\frac{\alpha}{2} \Gamma(\alpha / 2)(d-1)^{\alpha / 2} .
$$

Since $R(y) \geq 0, y \in \mathbb{H}^{d}$, and $R(x)=0$ we have

$$
\mathcal{A}_{t} R(x)=\frac{1}{\mathbb{E}^{x} \tau_{B(x, t)}}\left(\mathbb{E}^{x}\left[R\left(X_{\tau_{B(x, t)}}\right)\right]-R(x)\right)=\frac{1}{\mathbb{E}^{x} \tau_{B(x, t)}} \mathbb{E}^{x}\left[R\left(X_{\tau_{B(x, t)}}\right)\right] \geq 0 .
$$

In fact, by Eq. 7 ,

$$
\begin{aligned}
\mathcal{A}_{t} R(x)= & \frac{1}{\mathbb{E}^{x} \tau_{B(x, t)}} \mathbb{E}^{x}\left[R\left(X_{\left.\tau_{B(x, t)}\right)}, X_{\tau_{B(x, t)}} \in B(x, 2 t)\right]\right. \\
& +\frac{1}{\mathbb{E}^{x} \tau_{B(x, t)}} \int_{B(x, 2 t)^{c}} R(y) P_{t}(x, y) \mu(d y)=I_{1}+I_{2},
\end{aligned}
$$

where $P_{t}(x, y)=\int_{B(x, t)} v(w, y) G_{B(x, t)}(x, w) \mu(d w)$. We shall split the integral (20) into several parts. Let $F=B(A, R / 2) \cap H$, where $A=\left(\tilde{0}, e^{-R}\right)$ is the point at which $H$ 
and the ball $D=B\left(x_{0}, R\right)$ are tangent. Since $\sup _{u \in B(x, t)} v(u, y) \leq v\left(d_{\mathbb{H}^{d}}(y, x) / 2\right)$ for $y \in B^{c}(x, 2 t)$ then

$$
P_{t}(x, y)=\int_{B(x, t)} v(w, y) G_{B(x, t)}(x, w) \mu(d w) \leq \mathbb{E}^{x} \tau_{B(x, t)} v\left(d_{\mathbb{H}^{d}}(x, y) / 2\right) .
$$

This shows that

$$
\begin{aligned}
I_{2} \leq & \frac{1}{\mathbb{E}^{x} \tau_{B(x, t)}} \int_{\left\{y_{d}>1\right\}} R(y) P_{t}(x, y) \mu(d y)+\int_{F^{c} \cap\left\{y_{d} \leq 1\right\}} R(y) v\left(d_{\mathbb{H} d}(y, x) / 2\right) \mu(d y) \\
& +\int_{F} R(y) v\left(d_{\mathbb{H} d}(y, x) / 2\right) \mu(d y) \\
= & I_{2}^{(1)}+I_{2}^{(2)}+I_{2}^{(3)}
\end{aligned}
$$

Applying Eq. 19, we get $\int_{\left\{y_{d}>1\right\}} h(y) v(w, y) \mu(d y) \leq \frac{C}{R^{\alpha / 2}}$ for $w \in B(x, t)$, which combined with $R(y) \leq h(y)$ yields

$$
\begin{aligned}
I_{2}^{(1)} & =\frac{1}{\mathbb{E}^{x} \tau_{B(x, t)}} \int_{\left\{y_{d}>1\right\}} R(y)\left(\int_{B(x, t)} v(w, y) G_{B(x, t)}(x, w) \mu(d w)\right) \mu(d y) \\
& \leq \frac{1}{\mathbb{E}^{x} \tau_{B(x, t)}} \int_{B(x, t)} G_{B(x, t)}(x, w) \mu(d w)\left(\int_{\left\{y_{d}>1\right\}} h(y) v(w, y) \mu(d y)\right) \\
& \leq \frac{C}{R^{\alpha / 2}} .
\end{aligned}
$$

Since $R(y) \leq h(y) \leq R^{\alpha / 2}$ for $y_{d}<1$, we get by Lemma 3.1,

$$
\begin{aligned}
I_{2}^{(2)} & \leq R^{\alpha / 2} \int_{F^{c}} v\left(d_{\mathbb{H}^{d}}(x, y) / 2\right) \mu(d y) \leq R^{\alpha / 2} \int_{\left\{d_{\mathbb{H} d}(x, y) \geq R / 8\right\}} v\left(d_{\mathbb{H}^{d}}(x, y) / 2\right) \mu(d y) \\
& \leq C \frac{R^{\alpha / 2}}{R^{\alpha}}=\frac{C}{R^{\alpha / 2}} .
\end{aligned}
$$

Next, we estimate the integral $I_{2}^{(3)}$.

Let $y \in B\left(A^{*}, R / 2\right)$ be such that $\log y_{d}<-R / 2$, where $A^{*}=\left(\tilde{0}, e^{-R / 2}\right)$. Then by Lemma $6.1,3 \delta_{D}(y) \geq \delta_{H}(y)$ and by Lemma $6.3, \delta_{H}(y)-\delta_{D}(y) \leq 3 \frac{|\tilde{y}|^{2}}{R}$, hence by the Lagrange theorem we obtain

$$
\begin{aligned}
R(y)=h(y)-f(y) & \leq(\alpha / 2)\left(\delta_{B\left(x_{0}, R\right)}\right)^{\alpha / 2-1}\left(\delta_{H}(y)-\delta_{D}(y)\right) \\
& \leq(3 \alpha / 2)\left(\delta_{B\left(x_{0}, R\right)}(y)\right)^{\alpha / 2-1} \frac{|\tilde{y}|^{2}}{R} \\
& \leq C\left(\delta_{H}(y)\right)^{\alpha / 2-1} \frac{|\tilde{y}|^{2}}{R} .
\end{aligned}
$$

Hence, if $F_{1}=F \cap B\left(A^{*}, R / 2\right)$, then $F_{1} \subset\left\{\log y_{d}<-R / 2\right\}$ and we have

$$
\begin{aligned}
\int_{F_{1}} R(y) v\left(d_{\mathbb{H}^{d}}(y, x) / 2\right) \mu(d y) & \leq \int_{F_{1}} C\left(R+\log y_{d}\right)^{\alpha / 2-1} \frac{|\tilde{y}|^{2}}{R} v\left(d_{\mathbb{H}^{d}}(y, x) / 2\right) \mu(d y) \\
& =\int_{F_{1} \cap\left\{d_{\mathbb{H}^{d}}\left(y_{d}, x_{d}\right) \leq|\tilde{y}|\right\}}+\int_{F_{1} \cap\left\{d_{\mathbb{H}^{d}}\left(y_{d}, x_{d}\right) \geq|\tilde{y}|\right\}}=J_{1}+J_{2} .
\end{aligned}
$$

Here, with a slight abuse of notation, the hyperbolic distance $d_{\mathbb{H} d}\left(y_{d}, x_{d}\right)$ is understood as the hyperbolic distance between $\left(\tilde{0}, x_{d}\right)$ and $\left(\tilde{0}, x_{d}\right)$ which equals $\left|\log x_{d}-\log y_{d}\right|$. Recall that $\mu(d y)=\frac{d y_{d} d \tilde{y}}{y_{d}^{d}}$ is the volume measure. Below we use the following estimate: for 
$0<R<1$ there holds $\frac{1}{y_{d}^{d-1}}<e^{R(d-1)}<e^{d-1}, y \in F_{1}$. By Lemma 6.2, $|\tilde{y}| \leq 2 d_{\mathbb{H}^{d}}(y, x)$, hence $v\left(d_{\mathbb{H}^{d}}(y, x) / 2\right) \leq v(|\tilde{y}| / 4)$ and

$$
\begin{aligned}
J_{1} & \leq \int_{F_{1} \cap\left\{d_{\mathbb{H}^{d}}\left(y_{d}, x_{d}\right) \leq|\tilde{y}|\right\}} C\left(R+\log y_{d}\right)^{\alpha / 2-1} \frac{|\tilde{y}|^{2}}{R} v(|\tilde{y}| / 4) \frac{d y_{d} d \tilde{y}}{y_{d}^{d}} \\
& \leq e^{d-1} \int_{e^{-R}}^{e^{-R / 2}}\left(R+\log y_{d}\right)^{\alpha / 2-1}\left[\int_{d_{\mathbb{H}^{d}}\left(y_{d}, x_{d}\right)}^{R} \frac{\rho^{2}}{R} v(\rho / 4) \rho^{d-2} d \rho\right] \frac{d y_{d}}{y_{d}} .
\end{aligned}
$$

After changing variables $\log y_{d}+R=w$, the last integral equals to

$$
\frac{c}{R} \int_{0}^{R / 2} w^{\alpha / 2-1} \int_{\left|R+\log x_{d}-w\right|}^{R} v(\rho / 4) \rho^{d} d \rho d w .
$$

Let $w_{0}=R+\log x_{d}$. Using the estimate $v(\rho) \approx \rho^{-d-\alpha}, \rho<1$, given in Lemma 3.1, we get

$$
\begin{aligned}
J_{1} & \leq \frac{C}{R} \int_{0}^{R / 2} w^{\alpha / 2-1} \int_{\left\{\left|w_{0}-w\right| \leq \rho \leq R\right\}} \rho^{-\alpha} d \rho d w \\
& \leq \frac{C}{R} \int_{0}^{R / 2} w^{\alpha / 2-1}\left|w_{0}-w\right|^{1-\alpha} d w \\
& \leq \frac{C}{R^{\alpha / 2}} .
\end{aligned}
$$

Since $d_{\mathbb{H}^{d}}\left(y_{d}, x_{d}\right) \leq d_{\mathbb{H}^{d}}(y, x)$, we have $v\left(d_{\mathbb{H}^{d}}(y, x) / 2\right) \leq v\left(d_{\mathbb{H}^{d}}\left(y_{d}, x_{d}\right) / 2\right)$. Using again the estimate $\frac{1}{y_{d}^{d}} \leq e^{R d} \leq e^{d}$, we get

$$
\begin{aligned}
J_{2} & \leq \frac{C}{R} \int_{F_{1} \cap\left\{d_{\mathbb{H}^{d}}\left(y_{d}, x_{d}\right) \geq|\tilde{y}|\right\}}\left(R+\log y_{d}\right)^{\alpha / 2-1}|\tilde{y}|^{2} v\left(d_{\mathbb{H}^{d}}\left(y_{d}, x_{d}\right) / 2\right) d \tilde{y} d y_{d} \\
& \left.\leq \frac{C}{R} \int_{\left\{0 \leq \log y_{d}+R<R / 2\right\}}\left(R+\log y_{d}\right)^{\alpha / 2-1} v\left(d_{\mathbb{H}^{d}}\left(y_{d}, x_{d}\right) / 2\right) \int_{\left\{|\tilde{y}| \leq d_{\mathbb{H}} d\right.}\left(y_{d}, x_{d}\right)\right\} \\
& =\frac{C}{R} \int_{\left\{0 \leq \log y_{d}+R<R / 2\right\}}\left(R+\log y_{d}\right)^{\alpha / 2-1} v\left(d_{\mathbb{H}^{d}}\left(y_{d}, x_{d}\right) / 2\right) d_{\mathbb{H}^{d}}\left(y_{d}, x_{d}\right)^{d+1} d y_{d} \\
& =\frac{C}{2^{d+1} R} \int_{0}^{R / 2} w^{\alpha / 2-1} v\left(\left|w-w_{0}\right| / 2\right)\left|w-w_{0}\right|^{d+1} d w \\
& \leq \frac{C}{R} \int_{0}^{R / 2} w^{\alpha / 2-1}\left|w_{0}-w\right|^{1-\alpha} d w \\
& \leq \frac{C}{R^{\alpha / 2}},
\end{aligned}
$$

where we used the change of variables $\log y_{d}+R=w$ and next the estimate $v(\rho) \leq c \rho^{-d-\alpha}$ from Lemma 3.1. This concludes the estimate

$$
\int_{F_{1}} R(y) v\left(d_{\mathbb{H} d}(y, x) / 2\right) \mu(d y) \leq \frac{C}{R^{\alpha / 2}} .
$$

Next, we deal with the integral over $G=F \cap\left(B\left(A^{*}, R / 2\right)\right)^{c}$. Let $y=\left(y_{d}, \tilde{y}\right)$ with $d_{\mathbb{H}^{d}}\left(y, A^{*}\right)=R / 2$ such that $\log y_{d} \leq-R / 2$. Then by Eq. 1 ,

$$
\cosh (R / 2)=1+\frac{\left|A^{*}-y\right|^{2}}{2 y_{d} e^{-R / 2}}=1+\frac{\left|e^{-R / 2}-y_{d}\right|^{2}+|\tilde{y}|^{2}}{2 y_{d} e^{-R / 2}} .
$$


Solving this equation with respect to $y_{d}$ and denoting $|\tilde{y}|=\rho$ we obtain

$$
\begin{aligned}
y_{d}(\rho) & =\frac{e^{-R}+\rho^{2}}{1+e^{-R / 2}\left(\sqrt{\sinh ^{2} \frac{R}{2}-\rho^{2} e^{R}}-\sinh \frac{R}{2}\right)} \\
& =e^{-R} \frac{1+\rho^{2} e^{R}}{1+e^{-R / 2}\left(\sqrt{\sinh ^{2} \frac{R}{2}-\rho^{2} e^{R}}-\sinh \frac{R}{2}\right)}
\end{aligned}
$$

which is then used to show that (for $R<1$ ):

$$
\begin{aligned}
R+\log \left(y_{d}(\rho)\right) & =\log \frac{1+e^{R} \rho^{2}}{1+e^{-R / 2}\left(\sqrt{\sinh ^{2} \frac{R}{2}-\rho^{2} e^{R}}-\sinh \frac{R}{2}\right)} \\
& =\log \frac{1+e^{R} \rho^{2}}{1+e^{-R / 2}\left(\sinh \frac{R}{2}\right)\left(\sqrt{1-\frac{\rho^{2} e^{R}}{\sinh ^{2} \frac{R}{2}}}-1\right)} \\
& \leq \log \frac{1+\rho^{2} e^{R}}{1-\frac{\rho^{2} e^{R / 2}}{\sinh R / 2}} \\
& \leq C \frac{\rho^{2}}{R} .
\end{aligned}
$$

Again, by Lemma 6.2, $\frac{|\tilde{y}|}{2} \leq d_{\mathbb{H} d}(x, y)$ for $y \in G$, hence $v\left(d_{\mathbb{H} d}(x, y) / 2\right) \leq v(|\tilde{y}| / 4)$. After changing variables $z=R+\log y_{d}$ and applying the estimate $R+\log \left(y_{d}(\rho)\right) \leq C \frac{\rho^{2}}{R}$, we obtain

$$
\begin{aligned}
\int_{G} R(y) v\left(d_{\mathbb{H}^{d}}(x, y) / 2\right) \mu(d y) & \leq \int_{G} h(y) v\left(d_{\mathbb{H}^{d}}(x, y) / 2\right) \mu(d y) \\
& \leq C \int_{0}^{\frac{1-e^{-R}}{2}} \rho^{d-2} v\left(\frac{\rho}{4}\right) d \rho \int_{e^{-R}}^{y_{d}(\rho)}\left(R+\log y_{d}\right)^{\alpha / 2} \frac{d y_{d}}{y_{d}^{d}} \\
& =C e^{(d-1) R} \int_{0}^{\frac{1-e^{-R}}{2}} \rho^{d-2} v\left(\frac{\rho}{4}\right) d \rho \int_{0}^{R+\log \left(y_{d}(\rho)\right)} z^{\alpha / 2} e^{-(d-1) z} d z \\
& \leq C \int_{0}^{\frac{1-e^{-R}}{2}} \rho^{d-2} v\left(\frac{\rho}{4}\right)\left(R+\log y_{d}(\rho)\right)^{\alpha / 2}\left(R+\log y_{d}(\rho)\right) d \rho \\
& \leq C \int_{0}^{\frac{1-e^{-R}}{2}} \rho^{d-2} v\left(\frac{\rho}{4}\right)\left(\frac{\rho^{2}}{R}\right)^{\alpha / 2} \frac{\rho^{2}}{R} d \rho \\
& \leq \frac{C}{R^{1+\alpha / 2}} \int_{0}^{R} \rho^{d+\alpha} v(\rho / 4) d \rho \\
& \leq \frac{c}{R^{\alpha / 2}},
\end{aligned}
$$

where in the last step we used Lemma 3.1. Putting together all the estimates obtained above, we get that $I_{2} \leq \frac{C}{R^{\alpha / 2}}$. It remains to estimate

$$
I_{1}=\frac{1}{\mathbb{E}^{x} \tau_{B(x, t)}} \mathbb{E}^{x}\left[R\left(X\left(\tau_{B(x, t)}\right)\right) ; X\left(\tau_{B(x, t)}\right) \in B(x, 2 t)\right] .
$$


Note that by Lemma 6.1 and the choice of $t$, for $y \in B(x, 2 t)$, we have $\delta_{B\left(x_{0}, R\right)}(y) \geq$ $\delta_{H}(y) / 3 \geq(R-2 t+\log x) / 3 \geq t$, hence applying the Lagrange theorem and Lemma 6.3 we get

$$
\begin{aligned}
h(y)-f(y) & \leq(\alpha / 2) t^{\alpha / 2-1}\left(\delta_{H}(y)-\delta_{D}(y)\right) \\
& \leq C t^{\alpha / 2-1} \frac{|\tilde{y}|^{2}}{R} \\
& \leq C t^{\alpha / 2-1} \frac{\sinh ^{2} 2 t}{R} \\
& \approx \frac{t^{1+\alpha / 2}}{R}
\end{aligned}
$$

By Eq. $16, \mathbb{E}^{x} \tau_{B(x, t)} \approx t^{\alpha}$ and $t \leq R$, hence we have

$$
\frac{1}{\mathbb{E}^{x} \tau_{B(x, t)}} \mathbb{E}^{x}\left[R\left(X\left(\tau_{B(x, t)}\right)\right), X\left(\tau_{B(x, t)}\right) \in B(x, 2 t)\right] \leq c \frac{t^{1-\alpha / 2}}{R} \leq \frac{C}{R^{\alpha / 2}} .
$$

The proof is completed.

Acknowledgments We thank A. Stós for a helpful discussion on the subject of the paper. We also thank the Referee for very useful comments and suggestions.

Open Access This article is distributed under the terms of the Creative Commons Attribution 4.0 International License (http://creativecommons.org/licenses/by/4.0/), which permits unrestricted use, distribution, and reproduction in any medium, provided you give appropriate credit to the original author(s) and the source, provide a link to the Creative Commons license, and indicate if changes were made.

\section{References}

1. Bogdan, K., Grzywny, T., Ryznar, M.: Barriers, exit time and survival probability for unimodal Lévy processes. Probab. Theory Relat. Fields 162, 155-198 (2015)

2. Borodin, A.N., Salminen, P.: Handbook of Brownian Motion: Facts and Formulae, Second Edition. Birkhäuser (2002)

3. Blumenthal, R.M., Getoor, R.K., Ray, D.B.: On the distribution of first hits for the symmetric stable processes. Trans. Amer. Math. Soc. 99, 540-554 (1961)

4. Byczkowski, T., Małecki, J., Ryznar, M.: Bessel potentials, hitting distributions and Green functions. Trans. Amer. Math. Soc 361, 4871-4900 (2009)

5. Chen, Z.-Q., Kim, P., Song, R.: Dirichlet heat kernel estimates for $\Delta^{\alpha / 2}+\Delta^{\beta / 2}$. Illinois J. of Math. 54, 1357-1392 (2010)

6. Davies, E.B.: Heat Kernels And Spectral Theory. Cambridge University Press, Cambridge (1989)

7. Getoor, R.K.: Infnitely divisible probabilities on the hyperbolic plane. Pacific J. Math 11, 1287-1308 (1961)

8. Getoor, R.K.: First passage times for symmetric stable processes in space. Trans. Amer. Math. Soc 101, 75-90 (1961)

9. Graczyk, P., Stós, A.: Transition density estimates for stable processes on symmetric spaces. Pacific J. Math 217, 87-100 (2004)

10. Grigor'yan, A., Noguchi, M.: The heat kernel on hyperbolic space. Bulletin of LMS 30, 643-650 (1998)

11. Helgason, S.: Groups and Geometric Analysis. Academic Press, New York (1984)

12. Hunt, G.A.: Markov processes and potentials I and II. Illinois J. of Math. 1, 44-93, 316-369 (1957)

13. Ikeda, N., Watanabe, S.: On some relations between the harmonic measure and the Lévy measure for a certain class of Markov processes. J. Math. Kyoto Univ. 2, 79-95 (1962)

14. Kim, P., Mimica, A.: Green function estimates for subordinate Brownian motions: Stable and beyond. Trans. Amer. Math. Soc. 366, 4383-4422 (2014)

15. Kim, P., Song, R., Vondracek, Z.: Two-sided Green function estimates for killed subordinate Brownian motions. Proc. London Math. Soc 104, 927-958 (2012) 
16. Pyć, A., Serafin, G., Żak, T.: Supremum distribution of Bessel process of drifting Brownian motion. Probab. Math. Stat. 35, 201-222 (2015)

17. Ratcliffe, J.G.: Foundations of Hyperbolic Manifolds, Graduate Texts in Math, p. 149. Springer, New York (2006)

18. Stós, A.: Stable semigroups on homogeneous trees and hyperbolic spaces. Illinois J. Math 55, 14371454 (2011) 